\title{
Skin-related symptoms following exposure to recreational water: a systematic review and meta-analysis
}

\author{
Vincent Yau • Timothy J. Wade • Carol K. de Wilde • \\ John M. Colford Jr.
}

Received: 4 November 2008 / Revised: 17 February 2009 / Accepted: 5 March 2009 / Published online: 31 March 2009

(C) The Author(s) 2009. This article is published with open access at Springerlink.com

\begin{abstract}
Exposure to contaminated recreational waters (defined by levels of fecal and other types of indicator bacteria) is associated with adverse health outcomes. The principal health outcome studied previously has been gastrointestinal illness. Although many studies included reports of frequent skin complaints (e.g. rash or itch) following recreational water exposure, no systematic reviews have examined the association between indicator levels and skin-related symptoms. Twenty relevant peer-reviewed studies were identified. The relative risks (swimmers vs. nonswimmers) of skin-related symptoms among those exposed to recreational water with bacterial indicator concentrations above threshold levels were determined using meta-analysis. Similarly, the relative risks (swimmers vs. non-swimmers) of skin-related complaints after exposure to water with bacterial indicator concentrations below threshold levels were determined. The ratio of these odds ratios (ROR) was then computed for each indicator. The risk of skin-related symptoms was significantly elevated in marine water with high levels of total coliforms ROR 1.86, (95\% CI 1.21, 2.87); fe-
\end{abstract}

This document has been subjected to review by the National Health and Environmental Effects Research Laboratory and approved for publication. Approval does not signify that the contents reflect the views of the Environmental Protection Agency.

The authors would like to gratefully acknowledge Alfred P. Dufour for assistance in identifying references.

V. Yau $(\bowtie) \cdot$ C.K. de Wilde · J.M. Colford Jr.

School of Public Health, Division of Epidemiology,

University of California, Berkeley, CA, USA

e-mail: vincentmyau@gmail.com

T.J. Wade

United States Environmental Protection Agency,

National Health and Environmental Effects Research Laboratory,

Chapel Hill, NC, USA cal coliforms ROR 1.45 (95\% CI 1.02, 2.07); E. coli ROR 1.98, (95\% CI 1.43, 2.75); enterococci ROR 2.04 (95\% CI $1.34,3.09)$ and fecal streptococci ROR 1.70 (95\% CI 1.07, 2.71). However, no significant associations with water quality indicators were demonstrated for the freshwater indicators examined (total coliform, fecal coliform, E. coli). Swimmers exposed to marine water at high levels of several indicator bacteria experience a significant increase in skin-related symptoms compared to non-swimmers. This relationship was not demonstrated in freshwater settings.

Keywords Recreational water - Bacterial indicators . Rash $\cdot$ Skin disease $\cdot$ Marine water $\cdot$ Fresh water

\section{Introduction}

Health hazards associated with recreational water exposure have been a subject of study since the 1950s. In response to these concerns, the U.S. Environmental Protection Agency published guidelines for ambient water quality (U.S. EPA 1986). These guidelines recommended that for marine waters the geometric means of at least five samples taken over a 30 day period should not exceed 35 colony forming units of enterococcus per $100 \mathrm{~mL}$. For fresh recreational waters, the cut-off points were 33 colony forming units (cfu) of enterococcus per $100 \mathrm{~mL}$, and $126 \mathrm{cfu}$ of E. coli per $100 \mathrm{~mL}$ (U.S. EPA 1986; Wade et al. 2003).

These recommendations were based on associations between fecal indicator bacteria and gastrointestinal illnesses. Gastrointestinal illness, however, may not be the only adverse health outcome associated with exposure to recreational waters. Skin, respiratory, ear, and other ailments are potentially associated with exposure to contaminated waters (WHO 2001). Although several studies have included an 
examination of the association between recreational water exposure and skin outcomes, no previous review has summarized the evidence systematically. The primary goal of this investigation is to quantify the association between microbial indicators used to monitor recreational water quality and skin-related outcomes in non-outbreak conditions in both marine and freshwater settings.

\section{Methods}

\section{Literature search}

The literature search was done using five electronic databases: PUBMED (http://www.ncbi.nlm.nih.gov/pubmed/), BIOSIS (http://apps.isiknowledge.com/), Web of Science (http://apps.isiknowledge.com/), EMBASE (http:// openaccess.dialog.com/med/), and ProQuest Dissertation and Theses (http://proquest.umi.com/pqdweb) for all dates until August 2008. The search terms used included key words "water and health," "water and fecal," "water and feces," "water and indicator," "recreational water and health," as well as permutations of the above keywords. We also contacted experts in the field and reviewed the citations of relevant studies for other relevant studies.

After gathering all available studies, we reviewed the titles and abstracts and retained relevant ones for full-text review. Studies were retained if the abstract and title pertained to health effects with respect to swimming in fresh or salt waters, and if the abstract or title suggested that microbiological quality of the water was measured. Studies published in all languages were considered, as long as the title and abstract were available in English.

\section{Selection criteria}

Studies were included in this review if they met the following criteria:

Water exposure: Studies related to marine (ocean) or fresh (lakes, rivers) waters were included, but studies involving swimming pools and other treated bodies of water were excluded. All forms of water contact were included (swimming, sporting events, bathing, etc.).

Water quality measures: Studies had to include at least one numeric measure of the microbiologic quality of the water. Studies without quantitative measures of microbiologic water quality were excluded.

Health outcomes: In addition to a measure of microbial water quality, a measure of human health associated with microbial water quality had to be reported. After full-text review, if no measure of skin-related symptoms (irritation, rash, infection, itchiness, etc.) was reported, the study was excluded.
Study design: Because the purpose of this review was to determine the relationship between microbial indicators and skin-related outcomes under non-outbreak conditions, only studies that dealt with endemic situations were considered for this review. Studies were also required to report data on a control group (otherwise we could not calculate a measure of relative risk).

Study characteristics: If a publication was based on data that had been previously published, the most recent analysis was abstracted and the earlier publication was excluded. Also, only peer-reviewed publications were retained.

\section{Data abstraction}

The data were abstracted independently by two authors (C.K.d. and V.Y.). For each study, the following data were abstracted: microbial water quality measure (type and numerical value), water type (marine or fresh), population studied, geographic location, study size, study design, how skin symptoms were defined, covariates adjusted for, comparison group, information on swimming exposure (type and duration), relative risks, and confidence bounds. If a measure of relative risk was not reported, then data were abstracted and used to calculate the odds ratio and its $95 \%$ confidence interval. If a publication reported data from several study sites, or the same site over several years, data for each site were abstracted and treated as a separate study (Table 2). If a study did not report a measure of relative risk comparing swimmers to a non-swimmer comparison group, but did have a different comparison group (i.e., swimmers in waters with minimal contamination; Haile et al. 1999), those relative risks were extracted instead. Three indicators (total fungi, Candida, and enteroviruses) were excluded from analysis because too few studies $(<2)$ examined their relation to skin-related outcomes.

A total of eight microbial indicators were included as part of the marine water meta-analysis and six as part of the freshwater meta-analysis (Table 2). The indicators included in the marine water analysis were total coliform, fecal coliform, fecal streptococci, E. coli, enterococci, Klebsiella, P. aeruginosa, and staphylococci. For the freshwater analysis, total coliform, fecal coliform, fecal streptococci, E. coli, enterococci, and staphylococci were included. Indicators were selected for analysis if two or more studies examined the indicators in relation to skin-related outcomes. Even though some indicators are currently recommended for use (i.e., U.S. EPA recommends enterococci and E. coli for fresh waters) this is based on their association with gastrointestinal illnesses (Cabelli 1983), not other symptoms. Thus, both currently recommended indicators and indicators not in current use were included. 
Cut-off points for threshold values

Values to define high bacterial levels were obtained for total coliforms, fecal coliforms, fecal streptococci, and enterococci, and these cut-off points were analyzed to determine if there was an association between these levels and skinrelated health outcomes. The California State Water Resources Control Board (1990) recommended that a cut-off point of 10,000 cfu / $100 \mathrm{~mL}$ be used for total coliform in marine waters, as well as a cut-off point of $400 \mathrm{cfu} / 100$ $\mathrm{mL}$ for fecal coliforms (California State Water Resources Control Board 1990b). Haile et al. (1999) recommended a cut-off point of $35 \mathrm{cfu} / 100 \mathrm{~mL}$ for E. coli. Ogan (1994) recommended a cut-off point of $35 \mathrm{cfu} / 100 \mathrm{~mL}$ for fecal streptococci, and the EPA recommendation for a cutoff point using enterococci was $35 \mathrm{cfu} / 100 \mathrm{~mL}$ (U.S. EPA 1986). California cut-off points were not selected preferentially; rather, any cut-off points found were considered. Alternative cut-off points were not found, potentially because non-recommended indicators are not as well researched.

For freshwater indicators, cut-off points were also obtained from the literature. The California Department of Public Health (2000) recommended a fecal coliform cut-off point of $200 \mathrm{cfu} / 100 \mathrm{~mL}$. A cut-off point of 1,000 cfu / $100 \mathrm{~mL}$ was recommended by the San Diego Water Board (2007) for total coliform. For fecal streptococci, a cut-off point of $100 \mathrm{cfu} / 100 \mathrm{~mL}$ was recommended in Wiedenmann et al. (2006); however, no studies were identified with fecal streptococci levels higher than this cut-off, so no comparison could be made.

Cut-off points proposed by the EPA and WHO were chosen preferentially, but when there were no established guidelines, cut-off points recommended by the literature were used instead, due to convenience. In marine settings, the EPA provided a recommended cut-off point for enterococci, but for the remaining fresh and marine indicators, there were no WHO or EPA recommended values. No cut-off points were found at all for concentrations of Klebsiella, P. aeruginosa, or staphylococci in marine waters, and no recommended cut-off point was found for staphylococci concentrations in fresh waters. Thus, no ROR was calculated, and only odds ratios comparing swimmers to control groups were computed for these indicators through meta-analysis.

\section{Data analysis}

Separate analyses were used to examine each combination of microbial indicator and water type (marine and fresh). If a study reported microbial indicator values over a range, the median value of that range was used in our analyses. Exposure categories were formed by basing defining thresholds for high exposure based on cut-off points listed in U.S. EPA and WHO criteria or guidelines recommended for safe recreational water (U.S. EPA 1986;
WHO 2001), and if those were unavailable, cut-off points recommended by the literature were used (San Diego Water Board 2007; Haile et al. 1999; California Department of Public Health 2000; Wiedenmann et al. 2006; Ogan 1994; Wade et al. 2003).

If a study reported multiple relative risks from a single study site, the highest exposure measure and its relative risk was used for analysis, consistent with the prior work of Wade et al. (2003). This prevented a single study from receiving more weight solely because of the number of results presented. However, if a study reported findings from multiple, independent study sites, the microbial indicator level and relative risk from each study site was recorded and used for analysis. Also, if a certain beach was studied one year and then studied again on a subsequent year, those study results were recorded separately. One potential concern related to treating each site in a report as a separate observation lies in the fact that the findings from those sites might, theoretically, not be independent of each other. However, pooling such sites might not be appropriate either because of differences in the swimming populations that utilized those beaches and in the indicator levels that were present at the time. Rather than combining potentially different populations and sites, we analyzed them as separate observations (Table 2).

\section{Meta-analysis of study site results}

We calculated a summary relative risk of skin-related outcomes for each microbial indicator level (i.e., one odds ratio for swimmers vs. non-swimmers above the indicator cut-off point and one odds ratio for swimmers vs. non-swimmers below the cut-off point using fixed-effects models if no heterogeneity was present; otherwise, random-effects models were utilized). Heterogeneity of study results was assessed for each analysis using the Q statistic (DerSimonian and Laird 1986).

A binary variable was created to categorize the data into the sites with mean indicator levels below the cut-off point and those above. We then compared the odds ratio of skinrelated outcomes for exposure above the cut-off point to the odds ratio of skin-related outcomes below the cut-off point. The mean difference between the log relative risks was taken, and the null hypothesis being tested was that the difference was equal to zero. The difference was then exponentiated to create the ratio of odds ratios (ROR). A ROR above 1.0 suggests an increased risk of skin-related symptoms among those exposed to indicator levels above the cutoff as compared to those exposed to indicator levels below the cut-off point (Altman and Bland 2003). For example, if the OR for swimmers vs. non-swimmers was 5.0 above the indicator cut-off point and 2.0 below the cut-off point, then the ROR would be reported as $2.5(=5.0 / 2.0)$. 
Heterogeneity

Sources of heterogeneity that might explain the variability between the results of different studies were investigated by using a random-effects meta-regression model (Thompson and Sharp 1999). The outcome being modeled was the natural $\log$ of the relative risk for skin-related outcomes and predictor variables were indicator variables for whether or not a study adjusted for a particular covariate (available covariates were: gender, respondent, socioeconomic status, age, health or allergy history, visitor or native status, ethnicity, food consumption, knowledge of beach health hazards, place of residence, marital status, use of randomization, exposure activity at the beach, insect repellant use, sunblock use, physical weather and wave data, beach density, presence of animals or boats, and swimming history), study size, study type, and geographic location of the study. The final model was chosen by excluding covariates with $p$-values $>0.2$.

Analyses were performed using Stata 10.0 for the Macintosh (Stata Corporation 2007).

\section{Results}

A total of 3,468 titles and abstracts were reviewed for relevance, and 47 of these were retained for full text review. Of these, 20 studies (Table 1) were retained for final analysis.
Twenty-seven studies were excluded because three studies included no information or inadequate information on microbiological water quality (Amson 1991; New Jersey DoH 1988; Baylet and Sinegre 1984), 16 were excluded because skin outcome data was not reported or was not adequately reported (Balarajan et al. 1991; Bandaranayake et al. 1995; Bonilla et al. 2007; Cheung et al. 1991; Dufour 1984; Fattal et al. 1987; Fleisher et al. 1996; Harrington et al. 1993; Kocasoy 1989; McBride et al. 1998; Philipp et al. 1985; Seyfried et al. 1985b; Wiedenmann et al. 2006; Foulon et al. 1983; Kueh et al. 1995; Marino et al. 1995), one was excluded because the indicator used was not reported in any other study (Pilotto et al. 1997, cyanobacteria), three were excluded because they did not generate any relevant primary data (Burke 2002; Pruss 1998; Robinton 1966), two were excluded because the same results were published elsewhere (Haile 1996; Zmirou et al. 1990), and one was excluded because data on a control group was not reported (Stevenson 1953).

\section{Characteristics of included studies}

The 20 studies retained for inclusion in the meta-analysis had study populations ranging from 104 to 23,241 individuals. Nine of the 20 studies were conducted in freshwater settings, while the remaining 11 studies were conducted in marine water settings (Table 1). One publication, Cabelli (1983), reported data from two separate studies (one in Louisiana, USA, and one in Egypt).
Table 1 Final list of studies retained, by date of publication, location of study, marine or freshwater exposure, total study sample size, and study design

\begin{tabular}{lllll}
\hline Reference & Location & Water Type & Sample Size & Study Type \\
\hline Cabelli et al. (1979) & USA & Marine & 8073 & Prospective Cohort \\
Cabelli (1983) & USA/Egypt & Marine & $3778 / 23241$ & Prospective Cohort \\
Seyfried et al. (1985) & Canada & Fresh & 4537 & Prospective Cohort \\
Dewailly et al. (1986) & Canada & Fresh & 120 & Prospective Cohort \\
Ferley et al. (1989) & France & Fresh & 5737 & Retrospective Cohort \\
Cheung et al. (1990) & Hong Kong & Marine & 18741 & Prospective Cohort \\
Jones et al. (1991) & UK & Marine & 276 & Randomized Cohort \\
Alexander et al. (1992) & UK & Marine & 703 & Prospective Cohort \\
Fewtrell et al. (1992) & UK & Fresh & 516 & Prospective Cohort \\
Von Schirnding et al. (1992) & South Africa & Marine & 733 & Prospective Cohort \\
Charoenca and Fujioka (1995) & USA & Marine & 106 & Case-Control \\
Medema et al. (1995) & Netherlands & Fresh & 395 & Prospective Cohort \\
Van Asperen et al. (1997) & Netherlands & Fresh & 104 & Randomized Cohort \\
Haile et al. (1999) & USA & Marine & 10459 & Prospective Cohort \\
Prieto et al. (2001) & Spain & Marine & 1858 & Prospective Cohort \\
Lee et al. (2002) & Indonesia & Fresh & 435 & Cross-Sectional \\
Dwight et al. (2004) & USA & Marine & 1873 & Cross-Sectional \\
Wiedenmann et al. (2006) & Germany & Fresh & 1759 & Randomized Cohort \\
Colford et al. (2007) & USA & Marine & 8797 & Prospective Cohort \\
Wade et al. (2008) & USA & Fresh & 21015 & Prospective Cohort \\
\hline
\end{tabular}


Table 2 Six freshwater indicators and 8 marine indicators retained for final analysis. Studies indicate the number of publications dealing with that indicator and skin disease, and sites refer to the number of beaches/independent locations that were studied in those publications

\begin{tabular}{|c|c|c|c|}
\hline Freshwater Indicators & Studies & Sites & Authors/Sites* \\
\hline Fecal Coliform & 6 & 3 & $\begin{array}{l}\text { Ferley et al. (1989)—3 Sites } \\
\text { Lee et al. (2002)—1 Site } \\
\text { Dewailly et al. (1986)—1 Site } \\
\text { Fewtrell et al. (1992)—2 Sites } \\
\text { Medema et al. (1995)—1 Site } \\
\text { Seyfried et al. (1985)—1 Site }\end{array}$ \\
\hline Total Coliform & 2 & 4 & $\begin{array}{l}\text { Ferley et al. (1989)—3 Sites } \\
\text { Lee et al. (2002)—1 Site }\end{array}$ \\
\hline Fecal Streptococci & 4 & 7 & $\begin{array}{l}\text { Ferley et al. (1989)—3 Sites } \\
\text { Fewtrell et al. (1992)—2 Sites } \\
\text { Medema et al. (1995)—1 Site } \\
\text { Seyfried et al. (1985)—1 Site }\end{array}$ \\
\hline E. coli & 3 & 3 & $\begin{array}{l}\text { Medema et al. (1995) - 1 Site } \\
\text { Van Asperen et al. (1997)_-1 Site } \\
\text { Wiedenmann et al. (2006) -1 Site }\end{array}$ \\
\hline Enterococcus & 3 & 3 & $\begin{array}{l}\text { Wade et al. (2008) - } 1 \text { Site } \\
\text { Van Asperen et al. (1997)—1 Site } \\
\text { Wiedenmann et al. (2006) -1 Site }\end{array}$ \\
\hline Staphylococci & 3 & 4 & $\begin{array}{l}\text { Fewtrell et al. (1992)—2 Sites } \\
\text { Sevfr ed et al. (1985)—1 Site } \\
\text { Van Asperen et al. (1997)—1 Site }\end{array}$ \\
\hline Marine Indicators & Studies & Sites & Authors/Sites ${ }^{*}$ \\
\hline Enterococci & 6 & 25 & $\begin{array}{l}\text { Cheung et al. (1990)—9 Sites } \\
\text { Cabelli et al. (1979)—2 Sites } \\
\text { Cabelli (1983)—10 Sites } \\
\text { Haile et al. (1999)—1 Site } \\
\text { Colford et al. (2007)—1 Site } \\
\text { Von Schirnding et al. (1992)—2 Sites }\end{array}$ \\
\hline E. coli & 4 & 22 & $\begin{array}{l}\text { Cheung et al. (1990)—9 Sites } \\
\text { Cabelli et al. (1979)—2 Sites } \\
\text { Cabelli (1983)—10 Sites } \\
\text { Haile et al. (1999)—1 Site }\end{array}$ \\
\hline Total Coliforms & 7 & 9 & $\begin{array}{l}\text { Prieto et al. (2001)—1 Site } \\
\text { Jones et al. (1991)—1 Site } \\
\text { Cabelli et al. (1979)—2 Sites } \\
\text { Alexander et al. (1992)—1 Site } \\
\text { Haile et al. (1999)—1 Site } \\
\text { Colford et al. (2007)—1 Site } \\
\text { Dwight et al. (2004)—2 Sites }\end{array}$ \\
\hline Fecal Streptococci & 5 & 14 & $\begin{array}{l}\text { Cheung et al. (1990)—9 Sites } \\
\text { Prieto et al. (2001)—1 Site } \\
\text { Jones et al. (1991)—1 Site } \\
\text { Cabelli et al. (1979)—-2 Sites } \\
\text { Alexander et al. (1992)—1 Site }\end{array}$ \\
\hline
\end{tabular}

* Only distinct beaches or sites that were analyzed separately and for which a separate relative risk was reported were counted as a site 


\begin{tabular}{|c|c|c|c|}
\hline Marine Indicators & Studies & Sites & Authors/Sites ${ }^{*}$ \\
\hline Fecal Coliform & 8 & 18 & $\begin{array}{l}\text { Cheung et al. (1990)—9 Sites } \\
\text { Prieto et al. (2001)—1 Site } \\
\text { Jones et al. (1991)—1 Site } \\
\text { Cabelli et al. (1979)—2 Sites } \\
\text { Alexander et al. (1992)—1 Site } \\
\text { Haile et al. (1999)—1 Site } \\
\text { Colford et al. (2007)—1 Site } \\
\text { Von Schirnding et al. (1992)—2 Sites }\end{array}$ \\
\hline Klebsiella & 2 & 11 & $\begin{array}{l}\text { Cheung et al. (1990)—9 Sites } \\
\text { Cabelli et al. (1979)—2 Sites }\end{array}$ \\
\hline P. aeruginosa & 4 & 13 & $\begin{array}{l}\text { Cheung et al. (1990)—9 Sites } \\
\text { Prieto et al. (2001)—1 Site } \\
\text { Jones et al. (1991)—1 Site } \\
\text { Cabelli et al. (1979)—2 Sites }\end{array}$ \\
\hline Staphylococci & 3 & 11 & $\begin{array}{l}\text { Cheung et al. (1990)—9 Sites } \\
\text { Prieto et al. (2001)—1 Site } \\
\text { Charoenca and Fujioka (1995)—1 Site }\end{array}$ \\
\hline
\end{tabular}

* Only distinct beaches or sites that were analyzed separately and for which a separate relative risk was reported were counted as a site status, and history of river exposure was collected on the same day. The comparison groups were those with exposure to highly contaminated water vs. lower water contamination. For Dwight et al. (2004), surfers who surfed at least once a week were interviewed at two different beaches (one highly contaminated and one less contaminated) and were asked about symptoms in the past 3 months as well as exposure history for that time. Mean monthly indicator levels were provided by local health agencies.

With respect to randomized trials, Jones et al. (1991) randomly assigned individuals to swimming or non-swimming behavior. Skin-related symptoms were assessed 3 days and 3 weeks after exposure, and water quality was assessed the day of exposure. Van Asperen et al. (1997) also randomized individuals to swimming or non-swimming and assessed skin symptoms one week after exposure. Water quality was measured five minutes before exposure. Wiedenmann et al. (2006) was similar to both of the above: outcomes were measured one week after the study, and individuals were randomized to exposure or non-exposure.

One retrospective cohort study conducted by Ferley et al. (1989) collected data on health status and water exposure the week before. Water quality was assessed by collecting samples in advance of the health surveys. Samples were collected 2 days per week, and the concentrations measured on those days were extrapolated to the adjacent days.

The case-control study conducted by Charoenca and Fujioka (1995) measured water quality at various beaches and then enrolled patients with staphylococcal skin infections and determined their seawater contact 10 days before.

Eleven studies recruited both adults and children $(\mathrm{Ca}-$ belli et al. 1979; Cabelli 1983; Seyfried et al. 1985; Fer- 
Table 3 Exposure, outcome definitions, and outcome assessment methods for each study included in the meta-analysis. For some studies, limited information was available about outcome assessment methods

\begin{tabular}{|c|c|c|c|}
\hline Freshwater Studies & Exposure Definition & Outcome Definition & Outcome Assessment \\
\hline Seyfried et al. (1985) & Any contact & Skin rash, welts, boils & $\begin{array}{l}\text { Self-report symptoms, telephone } \\
\text { interview } 7-10 \text { days after, } \\
\text { questionnaire mailed if no contact }\end{array}$ \\
\hline Dewailly et al. (1986) & Windsurfers & $\begin{array}{l}\text { Infectious and allergenic skin } \\
\text { conditions }\end{array}$ & $\begin{array}{l}\text { Self-report symptoms, } \\
\text { questionnaire } 2 \text { days after }\end{array}$ \\
\hline Ferley et al. (1989) & Bathing & Skin infections & $\begin{array}{l}\text { Self-report symptoms, interviewed } \\
1 \text { week after }\end{array}$ \\
\hline Fewtrell et al. (1992) & Canoeists & Undefined skin symptoms & $\begin{array}{l}\text { Self-report symptoms, telephone } \\
\text { interview 5-7 days after, } \\
\text { questionnaire } 1 \text { month after }\end{array}$ \\
\hline Medema et al. (1995) & Triathalon-swim & Skin or mucosal symptoms & Self-report symptoms \\
\hline Van Asperen et al. (1997) & Head immersion & $\begin{array}{l}\text { Itchy skin, skin rash, present at } \\
\text { least } 2 \text { parts of the day in the } 2 \\
\text { days post trial }\end{array}$ & $\begin{array}{l}\text { Self-report symptoms, } \\
\text { questionnaire } 5 \text { days after }\end{array}$ \\
\hline Lee et al. (2002) & $\begin{array}{l}\text { Daily, some days, never exposure } \\
\text { to water }\end{array}$ & $\begin{array}{l}\text { Doctor inspection for skin } \\
\text { conditions }\end{array}$ & $\begin{array}{l}\text { Doctors assessed symptoms, day } \\
\text { of study }\end{array}$ \\
\hline Wiedenmann et al. (2006) & $\begin{array}{l}3 \text { head immersions, } 10 \text { minutes in } \\
\text { water }\end{array}$ & Skin infections or cutireactions & $\begin{array}{l}\text { Doctors assessed symptoms } 1 \\
\text { week after, questionnaire } 3 \text { weeks } \\
\text { after }\end{array}$ \\
\hline Wade et al. (2003) & Waist or higher immersion & Rash or itchy skin & $\begin{array}{l}\text { Self-report symptoms, telephone } \\
\text { interview 10-12 days after }\end{array}$ \\
\hline Marine Studies & Exposure Definition & Outcome Definition & Outcome Assessment \\
\hline Cabelli et al. (1979) & Head immersion, $>10 \mathrm{~min}$ in water & Itchy skin, rash, welts & $\begin{array}{l}\text { Self-report symptoms, telephone } \\
\text { interview 8-10 days after }\end{array}$ \\
\hline Cabelli (1983) & Head immersion & $\begin{array}{l}\text { Irritations and disturbances of the } \\
\text { skin }\end{array}$ & $\begin{array}{l}\text { US: Self-report symptoms, } \\
\text { telephone interview } 7-10 \text { days } \\
\text { after, Egypt: Self-report } \\
\text { symptoms, } 1 \text { week follow-up }\end{array}$ \\
\hline Cheung et al. (1990) & $\begin{array}{l}\text { Head immersion or water touching } \\
\text { face }\end{array}$ & $\begin{array}{l}\text { Rashes, skin symptoms exclusive } \\
\text { of sunburn }\end{array}$ & $\begin{array}{l}\text { Self-report symptoms, telephone } \\
\text { interview } 7 \text { days after }\end{array}$ \\
\hline Jones et al. (1991) & Head immersion & Skin rash & Self-report symptoms, 3 days after \\
\hline Alexander et al. (1992) & Any contact & Skin rash, itchy skin & $\begin{array}{l}\text { Self-report symptoms, telephone } \\
\text { and questionnaire } 10 \text { days after }\end{array}$ \\
\hline Von Schirnding et al. (1992) & Water contact beyond waist & Skin rash, itchy skin, welts & $\begin{array}{l}\text { Self-report symptoms, telephone } \\
\text { interview } 4 \text { days after }\end{array}$ \\
\hline Charoenca and Fujioka (1995) & $\begin{array}{l}\text { Seawater contact } 10 \text { days before } \\
\text { study }\end{array}$ & $\begin{array}{l}\text { Staphylococcal skin infections, } \\
\text { cultured }\end{array}$ & $\begin{array}{l}\text { Patients reporting to clinic with } \\
\text { skin infections recruited }\end{array}$ \\
\hline Haile et al. (1999) & Head immersion & Skin rash & $\begin{array}{l}\text { Self-report symptoms, telephone } \\
\text { interview 9-14 days after }\end{array}$ \\
\hline Prieto et al. (2001) & $\begin{array}{l}\text { Self report swimming activity, no } \\
\text { definition reported }\end{array}$ & Skin irritation, itching & $\begin{array}{l}\text { Self-report symptoms, telephone } \\
\text { interview } 7 \text { days after }\end{array}$ \\
\hline Dwight et al. (2004) & Surfers in polluted beaches & Self reported skin infection & Self-report symptoms, interview \\
\hline Colford et al. (2007) & Head or face under water & Skin rashes, infected cuts/scrapes & $\begin{array}{l}\text { Self-report symptoms, telephone } \\
\text { interview } 14 \text { days after }\end{array}$ \\
\hline
\end{tabular}

ley et al. 1989; Cheung et al. 1990; Von Schirnding et al. 1992; Haile et al. 1999; Prieto et al. 2001; Colford et al. 2007; Wiedenmann et al. 2006; Wade et al. 2008), while five studies only recruited adults (Jones et al. 1991; Dewailly et al. 1986; Fewtrell et al. 1992; Medema et al. 1995; Dwight et al. 2004). Only four studies focused only on children (Alexander et al. 1992; Lee et al. 2002; Van Asperen et al. 1997; Charoenca and Fujioka 1995). Definitions of outcomes and exposures for all the included studies are in Table 3.

Most studies used non-swimmers as the comparison group, but the chosen populations differed. Thirteen studies 
Table 4 Additional study information, by freshwater indicator. Water sampling method, laboratory analysis method, suspected contamination source, and comparison groups

\begin{tabular}{|c|c|c|c|c|c|}
\hline Freshwater Indicator & Study & Collection Method & Lab Method & Probable Source & Study Groups \\
\hline \multirow[t]{6}{*}{ Fecal Coliform } & Ferley et al. (1989) & $\begin{array}{l}2 \times \text { a week at } 5 \\
\text { beaches, } 30 \mathrm{~cm} \\
\text { depth }\end{array}$ & $\begin{array}{l}\text { Spread plate or } \\
\text { membrane filter } \\
\text { procedure with } \\
\text { Tergitol and TTC } \\
\text { agar, incubated }\end{array}$ & $\begin{array}{l}\text { Untreated urban } \\
\text { domestic sewage }\end{array}$ & $\begin{array}{l}\text { Swimmers vs. } \\
\text { Non-swimmers }\end{array}$ \\
\hline & Lee et al. (2002) & $\begin{array}{l}\text { Water sampled at } \\
\text { survey time }\end{array}$ & None Stated & $\begin{array}{l}\text { Pulp Mill, Treated } \\
\text { Waste, Point Source }\end{array}$ & $\begin{array}{l}\text { Village with high } \\
\text { pollution vs. Village } \\
\text { with low pollution }\end{array}$ \\
\hline & Dewailly et al. (1986) & $\begin{array}{l}\text { One day, } 8 \text { sites } \\
\text { sampled }\end{array}$ & None Stated & Sewage & $\begin{array}{l}\text { Windsurfers vs. } \\
\text { Non-water exposed }\end{array}$ \\
\hline & Fewtrell et al. (1992) & Day of activity & None Stated & $\begin{array}{l}\text { Several Upstream } \\
\text { Sewage } \\
\text { Treatment Plants }\end{array}$ & $\begin{array}{l}\text { Canoeists vs. } \\
\text { Non-canoeists }\end{array}$ \\
\hline & Medema et al. (1995) & $\begin{array}{l}\text { Samples of } 3 \text { sites, } \\
30 \mathrm{~cm} \text { below surface }\end{array}$ & $\begin{array}{l}\text { Dutch Standard } \\
\text { methods }\end{array}$ & None Stated & $\begin{array}{l}\text { Swimmers vs. } \\
\text { Non-swimmers }\end{array}$ \\
\hline & Seyfried et al. (1985) & $\begin{array}{l}\text { Sample Beaches } \\
2-3 \times \text { a day, water } \\
\text { and sediment at } \\
\text { depth of at least } 50 \\
\mathrm{~cm}\end{array}$ & $\begin{array}{l}\text { Water: MPN (Most } \\
\text { Probable Number) } \\
\text { using Standard } \\
\text { Methods }\end{array}$ & None Stated & $\begin{array}{l}\text { Swimmers vs. } \\
\text { Non-swimmers }\end{array}$ \\
\hline \multirow[t]{2}{*}{ Total Coliform } & Ferley et al. (1989) & $\begin{array}{l}2 \times \text { a week at } 5 \\
\text { beaches, } 30 \mathrm{~cm} \\
\text { depth }\end{array}$ & $\begin{array}{l}\text { Spread plate or } \\
\text { membrane filter } \\
\text { procedure with } \\
\text { Tergitol and TTC } \\
\text { agar, incubated }\end{array}$ & $\begin{array}{l}\text { Untreated urban } \\
\text { domestic sewage }\end{array}$ & $\begin{array}{l}\text { Swimmers vs. } \\
\text { Non-swimmers }\end{array}$ \\
\hline & Lee et al. (2002) & $\begin{array}{l}\text { Water sampled at } \\
\text { survey time }\end{array}$ & None Stated & $\begin{array}{l}\text { Pulp Mill, Treated } \\
\text { Waste, Point Source }\end{array}$ & $\begin{array}{l}\text { Village with high } \\
\text { pollution vs. Village } \\
\text { with low pollution }\end{array}$ \\
\hline \multirow[t]{4}{*}{ Fecal Streptococci } & Ferley et al. (1989) & $\begin{array}{l}2 \times \text { a week at } 5 \\
\text { beaches, } 30 \mathrm{~cm} \\
\text { depth }\end{array}$ & $\begin{array}{l}\text { Poured plates using } \\
\text { D. coccosel agar }\end{array}$ & $\begin{array}{l}\text { Untreated urban } \\
\text { domestic sewage }\end{array}$ & $\begin{array}{l}\text { Swimmers vs. } \\
\text { Non-Swimmers }\end{array}$ \\
\hline & Fewtrell et al. (1992) & Day of activity & None Stated & $\begin{array}{l}\text { Several Upstream } \\
\text { Sewage } \\
\text { Treatment Plants }\end{array}$ & $\begin{array}{l}\text { Canoeists vs. } \\
\text { Non-canoeists }\end{array}$ \\
\hline & Medema et al. (1995) & $\begin{array}{l}\text { Samples of } 3 \text { sites, } \\
30 \mathrm{~cm} \text { below surface }\end{array}$ & $\begin{array}{l}\text { Dutch Standard } \\
\text { methods }\end{array}$ & None Stated & $\begin{array}{l}\text { Swimmers vs. } \\
\text { Non-swimmers }\end{array}$ \\
\hline & Seyfried et al. (1985) & $\begin{array}{l}\text { Sample Beaches } \\
2-3 \times \text { a day, water } \\
\text { and sediment at } \\
\text { depth of at least } 50 \\
\mathrm{~cm}\end{array}$ & $\begin{array}{l}\text { Water: MPN (Most } \\
\text { Probable Number) } \\
\text { using Standard } \\
\text { Methods, and } \\
\text { membrane filter } \\
\text { m-Enterococcus } \\
\text { agar (Difco) }\end{array}$ & None Stated & $\begin{array}{l}\text { Swimmers vs. } \\
\text { Non-swimmers }\end{array}$ \\
\hline \multirow[t]{3}{*}{ E. coli } & Medema et al. (1995) & $\begin{array}{l}\text { Samples of } 3 \text { sites, } \\
30 \mathrm{~cm} \text { below surface }\end{array}$ & $\begin{array}{l}\text { Dutch Standard } \\
\text { methods }\end{array}$ & None Stated & $\begin{array}{l}\text { Swimmers vs. } \\
\text { Non-swimmers }\end{array}$ \\
\hline & Van Asperen et al. (1997) & $\begin{array}{l}\text { Day of exposure, } \\
\text { multiple sites, } 250 \\
\text { ml samples }\end{array}$ & $\begin{array}{l}\text { Dutch Standard } \\
\text { Methods }\end{array}$ & Treated sewage & $\begin{array}{l}\text { Primary School } \\
\text { children randomized }\end{array}$ \\
\hline & Wiedenmann et al. (2006) & $\begin{array}{l}\text { Sampled every } 20 \\
\text { min }\end{array}$ & $\begin{array}{l}\text { MUG Hydrolysis, } \\
\text { microtiter plate } \\
\text { method }\end{array}$ & $\begin{array}{l}\text { Treated and } \\
\text { untreated municipal } \\
\text { sewage, agricultural } \\
\text { run-off, waterfowl } \\
\text { contamination }\end{array}$ & $\begin{array}{l}\text { Randomized bathers } \\
\text { vs. non-bathers }\end{array}$ \\
\hline
\end{tabular}




\begin{tabular}{|c|c|c|c|c|c|}
\hline Freshwater Indicator & Study & Collection Method & Lab Method & Probable Source & Study Groups \\
\hline \multirow[t]{3}{*}{ Enterococcus } & Wade et al. (2008) & $\begin{array}{l}\text { Samples shin and } \\
\text { waist deep }\end{array}$ & $\begin{array}{l}\text { EPA Membrane } \\
\text { Filtration Method } \\
1600 \text {, and QPCR }\end{array}$ & $\begin{array}{l}\text { Treated Sewage } \\
\text { (Point Source) }\end{array}$ & $\begin{array}{l}\text { Swimmers vs. } \\
\text { Non-swimmers }\end{array}$ \\
\hline & Van Asperen et al. (1997) & $\begin{array}{l}\text { Day of exposure, } \\
\text { multiple sites, } 250 \\
\text { ml samples }\end{array}$ & $\begin{array}{l}\text { Dutch Standard } \\
\text { Methods }\end{array}$ & Treated sewage & $\begin{array}{l}\text { Primary School } \\
\text { children randomized }\end{array}$ \\
\hline & Wiedenmann et al. (2006) & $\begin{array}{l}\text { Sampled every } 20 \\
\text { min }\end{array}$ & $\begin{array}{l}\text { MUD Hydrolysis } \\
\text { and formazan } \\
\text { formation }\end{array}$ & $\begin{array}{l}\text { Treated and } \\
\text { untreated municipal } \\
\text { sewage, agricultural } \\
\text { run-off, waterfowl } \\
\text { contamination }\end{array}$ & $\begin{array}{l}\text { Randomized bathers } \\
\text { vs. non-bathers }\end{array}$ \\
\hline \multirow[t]{3}{*}{ Staphylococci } & Fewtrell et al. (1992) & Day of activity & None Stated & $\begin{array}{l}\text { Several Upstream } \\
\text { Sewage } \\
\text { Treatment Plants }\end{array}$ & $\begin{array}{l}\text { Canoeists vs. } \\
\text { Non-canoeists }\end{array}$ \\
\hline & Seyfried et al. (1985) & $\begin{array}{l}\text { Sample Beaches } \\
2-3 \times \text { a day, water } \\
\text { and sediment at } \\
\text { depth of at least } 50 \\
\mathrm{~cm}\end{array}$ & $\begin{array}{l}\text { Water: Gelman } \\
\text { Filters, incubated on } \\
\text { Vogel-Johnson agar, } \\
\text { Sand: Enrich in } \\
\text { m-Staphylococcus } \\
\text { broth, spread on } \\
\text { Vogel-Johnson agar }\end{array}$ & None Stated & $\begin{array}{l}\text { Swimmers vs. } \\
\text { Non-swimmers }\end{array}$ \\
\hline & Van Asperen et al. (1997) & $\begin{array}{l}\text { Day of exposure, } \\
\text { multiple sites, } 250 \\
\text { ml samples }\end{array}$ & $\begin{array}{l}\text { Dutch Standard } \\
\text { Methods }\end{array}$ & Treated sewage & $\begin{array}{l}\text { Primary School } \\
\text { children randomized }\end{array}$ \\
\hline
\end{tabular}

chose to use beach-goers who had no water exposure (Cabelli et al. 1979; Cabelli 1983; Cheung et al. 1990; Prieto et al. 2001; Seyfried et al. 1985; Von Schirnding et al. 1992; Colford et al. 2007; Alexander et al. 1992; Ferley et al. 1989; Jones et al. 1991; Wade et al. 2008; Van Asperen et al. 1997; Wiedenmann et al. 2006), while three studies used employees at a sporting event or athletes with no water exposure at the same sporting venue (Medema et al. 1995; Fewtrell et al. 1992; Dewailly et al. 1986). Haile et al. (1999), Lee et al. (2002), and Dwight et al. (2004) used exposure to less contaminated waters as a comparison group for individuals exposed to highly contaminated waters.

\section{Exposure assessment and definitions}

If studies did not report direct observation of water exposure, it was assumed that self-report was used instead. For fifteen of the twenty studies, exposure was determined by self-report (Cabelli et al. 1979; Cabelli 1983; Seyfried et al. 1985; Dewailly et al. 1986; Ferley et al. 1989; Cheung et al. 1990; Alexander et al. 1992; Von Schirnding et al. 1992; Haile et al. 1999; Prieto et al. 2001; Lee et al. 2002; Colford et al. 2007; Dwight et al. 2004; Charoenca and Fujioka 1995; Wade et al. 2008). The definition of exposure differed from study to study. The most common definition was head immersion or facial contact (Cabelli et al. 1979; Cabelli 1983; Cheung et al. 1990; Haile et al. 1999; Jones et al. 1991;
Colford et al. 2007). The next most common definition was any contact with the water (Alexander et al. 1992; Seyfried et al. 1985; Von Schirnding et al. 1992; Ferley et al. 1989). Three studies defined exposure as participation in a water-related sporting event (Medema et al. 1995; Fewtrell et al. 1992; Dewailly et al. 1986). See Table 3 for more detailed definitions.

Meta-analysis of study site results: marine water

The results of this analysis indicated that for all bacterial indicators tested, the odds ratios (of illness in swimmers vs. non-swimmers) at sites with low indicator levels were significantly smaller than odds ratios at sites with elevated indicator levels. The ROR comparing the odds ratios among swimmers in waters with high concentrations of enterococci vs. the odds ratio among swimmers in water with low concentrations was 2.04 (95\% CI 1.34-3.09). For total coliform, the ROR was 1.86 (95\% CI 1.21-2.87). Studies with fecal coliform levels above $400 \mathrm{cfu} / 100 \mathrm{~mL}$ had odds ratios that were 1.45 times larger than studies with lower indicator levels (95\% CI 1.02-2.07). For E. coli, the ROR was 1.96 (95\% CI 1.38-2.79). Studies with elevated fecal streptococci had an elevated odds ratio that was significantly different than a ROR of 1 (ROR $=1.70,95 \%$ CI 1.07-2.71) (Fig. 1). 
Table 5 Additional study information, by marine indicator. Water sampling method, laboratory analysis method, suspected contamination source, and comparison groups

\begin{tabular}{|c|c|c|c|c|c|}
\hline Marine Indicator & Study & Collection Method & Lab Method & Probable Source & Study Groups \\
\hline \multirow[t]{6}{*}{ Enterococci } & Cheung et al. (1990) & $\begin{array}{l}3 \text { samples per } \\
\text { beach, } 1 \mathrm{~m} \text { deep }\end{array}$ & $\begin{array}{l}\text { Membrane } \\
\text { filtration, incubated } \\
\text { on media }\end{array}$ & $\begin{array}{l}\text { Human sewage } \\
\text { discharge, } \\
\text { stormdrains, } \\
\text { livestock waste }\end{array}$ & $\begin{array}{l}\text { Swimmers vs. } \\
\text { non-swimmers }\end{array}$ \\
\hline & Cabelli et al. (1979) & $\begin{array}{l}\text { Sample several } \\
\text { times per day, chest } \\
\text { depth } 4 \text { in below } \\
\text { surface }\end{array}$ & Membrane filter & Sewage & $\begin{array}{l}\text { Swimmers vs. } \\
\text { Non-swimmers }\end{array}$ \\
\hline & Cabelli (1983) & $\begin{array}{l}\text { Multiple samples, } \\
\text { Chest Depth, just } \\
\text { below surface }\end{array}$ & $\begin{array}{l}\text { Membrane Filter, } \\
\text { mE medium }\end{array}$ & $\begin{array}{l}\text { US: None Stated, } \\
\text { Egypt: Raw sewage }\end{array}$ & $\begin{array}{l}\text { Swimmers vs. } \\
\text { Non-swimmers }\end{array}$ \\
\hline & Haile et al. (1999) & $\begin{array}{l}\text { Daily ankle depth } \\
\text { samples }\end{array}$ & Membrane filtration & $\begin{array}{l}\text { Storm Drain } \\
\text { Run-off }\end{array}$ & $\begin{array}{l}\text { Swimmers in more } \\
\text { polluted water vs. } \\
\text { swimmers in less } \\
\text { polluted water }\end{array}$ \\
\hline & Colford et al. (2007) & $\begin{array}{l}\text { Daily, hourly } \\
\text { sampling }\end{array}$ & $\begin{array}{l}\text { Membrane } \\
\text { Filtration, } \\
\text { chromogenic } \\
\text { substrate method, } \\
\text { and qPCR }\end{array}$ & $\begin{array}{l}\text { Non-point source, } \\
\text { human } \\
\text { contamination } \\
\text { minimal }\end{array}$ & $\begin{array}{l}\text { Swimmers vs. } \\
\text { non-swimmers }\end{array}$ \\
\hline & Von Schirnding et al. (1992) & $\begin{array}{l}\text { Day of trial, } \\
\text { samples before and } \\
\text { during trial }\end{array}$ & $\begin{array}{l}\text { Standard membrane } \\
\text { filtration methods }\end{array}$ & $\begin{array}{l}\text { Septic tank } \\
\text { overflows, } \\
\text { stormwater run-off, } \\
\text { fecal contamination } \\
\text { in river water }\end{array}$ & $\begin{array}{l}\text { Swimmers vs. } \\
\text { Non-swimmers }\end{array}$ \\
\hline \multirow[t]{4}{*}{ E. coli } & Cheung et al. (1990) & $\begin{array}{l}3 \text { samples per } \\
\text { beach, } 1 \mathrm{~m} \text { deep }\end{array}$ & $\begin{array}{l}\text { Membrane } \\
\text { filtration, incubated } \\
\text { on media }\end{array}$ & $\begin{array}{l}\text { Human sewage } \\
\text { discharge, } \\
\text { stormdrains, } \\
\text { livestock waste }\end{array}$ & $\begin{array}{l}\text { Swimmers vs. } \\
\text { Non-swimmers }\end{array}$ \\
\hline & Cabelli et al. (1979) & $\begin{array}{l}\text { Sample several } \\
\text { times per day, chest } \\
\text { depth } 4 \text { in below } \\
\text { surface }\end{array}$ & $\mathrm{mC}$ & Sewage & $\begin{array}{l}\text { Swimmers vs. } \\
\text { Non-swimmers }\end{array}$ \\
\hline & Cabelli (1983) & $\begin{array}{l}\text { Multiple samples, } \\
\text { Chest Depth, just } \\
\text { below surface }\end{array}$ & $\begin{array}{l}\text { Membrane } \\
\text { Filtration, mTEC } \\
\text { medium }\end{array}$ & $\begin{array}{l}\text { US: None Stated, } \\
\text { Egypt: Raw sewage }\end{array}$ & $\begin{array}{l}\text { Swimmers vs. } \\
\text { Non-swimmers }\end{array}$ \\
\hline & Haile et al. (1999) & $\begin{array}{l}\text { Daily ankle depth } \\
\text { samples }\end{array}$ & $\begin{array}{l}\text { Membrane } \\
\text { filtration, Hach } \\
\text { Method } 10029\end{array}$ & $\begin{array}{l}\text { Storm Drain } \\
\text { Run-off }\end{array}$ & $\begin{array}{l}\text { Swimmers in more } \\
\text { polluted water vs. } \\
\text { swimmers in less } \\
\text { polluted water }\end{array}$ \\
\hline
\end{tabular}

Meta-analysis of study site results: fresh water

Analyses of the cut-off points for fecal coliform and total coliform revealed no significant associations. For total coliform, the ROR was 1.17 (95\% CI 0.75-1.84). For fecal coliform, the ROR was 1.69 (95\% CI 0.88-3.27). The same conclusion was reached with $E$. coli as well $(\mathrm{ROR}=0.62$, 95\% CI 0.03-13.55), though the number of sites included was small $(n=3)$.

Meta-analysis of indicators without RORs: marine and fresh water

Three marine indicators (Klebsiella, P. aeruginosa, and staphylococci) and one freshwater indicator (staphylococci) had no recommended cut-off point, and so no ROR was calculated. However, a simple regression was used to determine if there was a linear relationship between concentration level of the indicator and the study OR. For Klebsiella, the OR associated with a one hundred cfu increase in concentration per $100 \mathrm{~mL}$ was 1.16 (95\% CI 0.98-1.37). For P. aeruginosa, the OR was 1.28 (95\% CI 0.30-5.44). For marine staphylococci, the OR was 1.02 (95\% CI 0.97-1.06). For freshwater staphylococci, the OR was 1.74 (95\% CI $0.17-$ 17.72).

One freshwater indicator, enterococcus, had a recommended cut-off point, but all available studies had indicator concentrations above the cut-off point. For a $100 \mathrm{cfu} /$ $100 \mathrm{~mL}$ increase in enterococcus concentrations in freshwa- 


\begin{tabular}{|c|c|c|c|c|c|}
\hline Marine Indicator & Study & Collection Method & Lab Method & Probable Source & Study Groups \\
\hline \multirow[t]{7}{*}{ Total Coliforms } & Prieto et al. (2001) & $30 \mathrm{~cm}$ below surface & Standard Methods & Sewage Systems & $\begin{array}{l}\text { Swimmers vs. } \\
\text { Non-Swimmers }\end{array}$ \\
\hline & Jones et al. (1991) & $\begin{array}{l}\text { Sampled every } 20 \\
\text { min at surf, } 30 \mathrm{~cm} \text {, } \\
\text { chest depth, and } 50 \\
\mathrm{~m} \text { off-shore. }\end{array}$ & None Stated & None Stated & $\begin{array}{l}\text { Bather vs. } \\
\text { Non-bather } \\
\text { Randomized }\end{array}$ \\
\hline & Cabelli et al. (1979) & $\begin{array}{l}\text { Sample several } \\
\text { times per day, chest } \\
\text { depth } 4 \text { in below } \\
\text { surface }\end{array}$ & $\begin{array}{l}\text { Most Probable } \\
\text { Number, mC } \\
\text { procedure }\end{array}$ & Sewage & $\begin{array}{l}\text { Swimmers vs. } \\
\text { Non-swimmers }\end{array}$ \\
\hline & Alexander et al. (1992) & $\begin{array}{l}2 \text { samples at waist } \\
\text { depth }\end{array}$ & Standard Methods & Sewage & $\begin{array}{l}\text { Swimmers vs. } \\
\text { Non-swimmers }\end{array}$ \\
\hline & Haile et al. (1999) & $\begin{array}{l}\text { Daily ankle depth } \\
\text { samples }\end{array}$ & $\begin{array}{l}\text { Membrane } \\
\text { Filtration }\end{array}$ & $\begin{array}{l}\text { Storm Drain } \\
\text { Run-off }\end{array}$ & $\begin{array}{l}\text { Swimmers in more } \\
\text { polluted water vs. } \\
\text { swimmers in less } \\
\text { polluted water }\end{array}$ \\
\hline & Colford et al. (2007) & $\begin{array}{l}\text { Daily, hourly } \\
\text { sampling }\end{array}$ & $\begin{array}{l}\text { Membrane } \\
\text { Filtration and } \\
\text { chromogenic } \\
\text { substrate method }\end{array}$ & $\begin{array}{l}\text { Non-point source, } \\
\text { human } \\
\text { contamination } \\
\text { minimal }\end{array}$ & $\begin{array}{l}\text { Swimmers vs } \\
\text { Non-swimmers }\end{array}$ \\
\hline & Dwight et al. (2004) & None Stated & None Stated & $\begin{array}{l}\text { Untreated Urban } \\
\text { Run-off (non-point } \\
\text { source) }\end{array}$ & $\begin{array}{l}\text { Polluted vs. } \\
\text { Non-polluted beach }\end{array}$ \\
\hline \multirow[t]{5}{*}{ Fecal Streptococci } & Cheung et al. (1990) & $\begin{array}{l}3 \text { samples per } \\
\text { beach, } 1 \mathrm{~m} \text { deep }\end{array}$ & $\begin{array}{l}\text { Membrane } \\
\text { filtration, incubated } \\
\text { on media }\end{array}$ & $\begin{array}{l}\text { Human sewage } \\
\text { discharge, } \\
\text { stormdrains, } \\
\text { livestock waste }\end{array}$ & $\begin{array}{l}\text { Swimmers vs. } \\
\text { Non-swimmers }\end{array}$ \\
\hline & Prieto et al. (2001) & $30 \mathrm{~cm}$ below surface & Standard Methods & Sewage Systems & $\begin{array}{l}\text { Swimmers vs. } \\
\text { Non-swimmers }\end{array}$ \\
\hline & Jones et al. (1991) & $\begin{array}{l}\text { Sampled every } 20 \\
\text { min at surf, } 30 \mathrm{~cm} \text {, } \\
\text { chest depth, and } 50 \\
\mathrm{~m} \text { off-shore }\end{array}$ & None Stated & None Stated & $\begin{array}{l}\text { Bather vs. } \\
\text { Non-bather } \\
\text { Randomized }\end{array}$ \\
\hline & Cabelli et al. (1979) & $\begin{array}{l}\text { Sample several } \\
\text { times per day, chest } \\
\text { depth } 4 \text { in below } \\
\text { surface }\end{array}$ & $\mathrm{mSD}$ & Sewage & $\begin{array}{l}\text { Swimmers vs. } \\
\text { Non-swimmers }\end{array}$ \\
\hline & Alexander et al. (1992) & $\begin{array}{l}2 \text { samples at waist } \\
\text { depth }\end{array}$ & Standard Methods & Sewage & $\begin{array}{l}\text { Swimmers vs. } \\
\text { Non-swimmers }\end{array}$ \\
\hline
\end{tabular}

ter settings, the OR was 0.88 (95\% CI $0.57-1.36$ ). A similar situation occurred with freshwater streptococci, but instead all studies had indicator concentrations lower than the recommended $100 \mathrm{cfu} / 100 \mathrm{~mL}$ cut-off point. A linear regression was performed, and the OR associated with a 100 cfu / $100 \mathrm{~mL}$ increase in concentration was 3.83 (95\% CI 0.60-24.39). Linear regression was chosen in lieu of other models mainly because the data were relatively sparse, and thus it was not obvious if the data were clearly linear or non-linear. While more complicated splines and exponential models could have been fit, the interpretation of the coefficients of these models would have been more difficult.

\section{Heterogeneity}

Heterogeneity was detected in several of the analyses $(p<0.2)$, and to explore sources of heterogeneity, metaregression was used. Factors that were considered were adjustment by the authors for any confounders, or adjustment for a variety of confounders (gender, respondent to survey, socioeconomic status (SES), age, history of health and allergies, visitor or native status, ethnicity, food consumption, knowledge of beach hazards, place of residence, marital status, exposure activities at the beach, insect repellant use, sunblock use, physical and weather data, density of individuals at the beach, presence of boats or animals, and swimming history). These covariates were coded as in- 


\begin{tabular}{|c|c|c|c|c|c|}
\hline Marine Indicator & Study & Collection Method & Lab Method & Probable Source & Study Groups \\
\hline \multirow[t]{8}{*}{ Fecal Coliform } & Cheung et al. (1990) & $\begin{array}{l}3 \text { samples per } \\
\text { beach, } 1 \text { m deep }\end{array}$ & $\begin{array}{l}\text { Membrane } \\
\text { filtration, incubated } \\
\text { on media }\end{array}$ & $\begin{array}{l}\text { Human sewage } \\
\text { discharge, } \\
\text { stormdrains, } \\
\text { livestock waste }\end{array}$ & $\begin{array}{l}\text { Swimmers vs. } \\
\text { Non-swimmers }\end{array}$ \\
\hline & Prieto et al. (2001) & $30 \mathrm{~cm}$ below surface & Standard Methods & Sewage Systems & $\begin{array}{l}\text { Swimmers vs. } \\
\text { Non-Swimmers }\end{array}$ \\
\hline & Jones et al. (1991) & $\begin{array}{l}\text { Sampled every } 20 \\
\text { min at surf, } 30 \mathrm{~cm} \text {, } \\
\text { chest depth, and } 50 \\
\mathrm{~m} \text { off-shore }\end{array}$ & None Stated & None Stated & $\begin{array}{l}\text { Bather vs. } \\
\text { Non-bather } \\
\text { Randomized }\end{array}$ \\
\hline & Cabelli et al. (1979) & $\begin{array}{l}\text { Sample several } \\
\text { times per day, chest } \\
\text { depth } 4 \text { in below } \\
\text { surface }\end{array}$ & $\begin{array}{l}\text { Most Probable } \\
\text { Number }\end{array}$ & Sewage & $\begin{array}{l}\text { Swimmers vs. } \\
\text { Non-swimmers }\end{array}$ \\
\hline & Alexander et al. (1992) & $\begin{array}{l}2 \text { samples at waist } \\
\text { depth }\end{array}$ & Standard Methods & Sewage & $\begin{array}{l}\text { Swimmers vs. } \\
\text { Non-swimmers }\end{array}$ \\
\hline & Haile et al. (1999) & $\begin{array}{l}\text { Daily ankle depth } \\
\text { samples }\end{array}$ & $\begin{array}{l}\text { Membrane } \\
\text { Filtration }\end{array}$ & $\begin{array}{l}\text { Storm Drain } \\
\text { Run-off }\end{array}$ & $\begin{array}{l}\text { Swimmers in more } \\
\text { polluted water vs. } \\
\text { swimmers in less } \\
\text { polluted water }\end{array}$ \\
\hline & Colford et al. (2007) & $\begin{array}{l}\text { Daily, hourly } \\
\text { sampling }\end{array}$ & $\begin{array}{l}\text { Membrane } \\
\text { Filtration and } \\
\text { chromogenic } \\
\text { substrate method }\end{array}$ & $\begin{array}{l}\text { Non-point source, } \\
\text { human } \\
\text { contamination } \\
\text { minimal }\end{array}$ & $\begin{array}{l}\text { Swimmers vs. } \\
\text { Non-swimmers }\end{array}$ \\
\hline & Von Schirnding et al. (1992) & $\begin{array}{l}\text { Day of trial, } \\
\text { samples before and } \\
\text { during trial }\end{array}$ & $\begin{array}{l}\text { Standard membrane } \\
\text { filtration methods }\end{array}$ & $\begin{array}{l}\text { Septic tank } \\
\text { overflows, } \\
\text { stormwater run-off, } \\
\text { fecal contamination } \\
\text { in river water }\end{array}$ & $\begin{array}{l}\text { Swimmers vs. } \\
\text { Non-swimmers }\end{array}$ \\
\hline \multirow[t]{2}{*}{ Klebsiella } & Cheung et al. (1990) & $\begin{array}{l}3 \text { samples per } \\
\text { beach, } 1 \mathrm{~m} \text { deep }\end{array}$ & $\begin{array}{l}\text { Membrane } \\
\text { filtration, incubated } \\
\text { on media }\end{array}$ & $\begin{array}{l}\text { Human sewage } \\
\text { discharge, } \\
\text { stormdrains, } \\
\text { livestock waste }\end{array}$ & $\begin{array}{l}\text { Swimmers vs. } \\
\text { Non-swimmers }\end{array}$ \\
\hline & Cabelli et al. (1979) & $\begin{array}{l}\text { Sample several } \\
\text { times per day, chest } \\
\text { depth } 4 \text { in below } \\
\text { surface }\end{array}$ & $\mathrm{mC}$ procedure & Sewage & $\begin{array}{l}\text { Swimmers vs. } \\
\text { Non-swimmers }\end{array}$ \\
\hline \multirow[t]{3}{*}{ Staphylococci } & Cheung et al. (1990) & $\begin{array}{l}3 \text { samples per } \\
\text { beach, } 1 \mathrm{~m} \text { deep }\end{array}$ & $\begin{array}{l}\text { Membrane } \\
\text { filtration, incubated } \\
\text { on media }\end{array}$ & $\begin{array}{l}\text { Human sewage } \\
\text { discharge, } \\
\text { stormdrains, } \\
\text { livestock waste }\end{array}$ & $\begin{array}{l}\text { Swimmers vs. } \\
\text { Non-swimmers }\end{array}$ \\
\hline & Prieto et al. (2001) & $30 \mathrm{~cm}$ below surface & Standard Methods & Sewage Systems & $\begin{array}{l}\text { Swimmers vs. } \\
\text { Non-swimmers }\end{array}$ \\
\hline & Charoenca and Fujioka (1995) & None Stated & $\begin{array}{l}\text { Gelman membrane } \\
\text { filtration, } \\
\text { Vogel-Johnson } \\
\text { Medium used with } \\
\text { incubation }\end{array}$ & None Stated & $\begin{array}{l}\text { swimmers at } \\
\text { polluted vs. less } \\
\text { polluted }\end{array}$ \\
\hline
\end{tabular}

dicator variables, with a " 1 " value indicating that the study adjusted for that covariate, and a " 0 " value for studies that did not adjust for that covariate.

For freshwater settings, the sources of heterogeneity for the fecal coliform meta-analysis were retrospective cohort study design $(\mathrm{OR}=2.42,1.08-5.41)$ and gender
$(\mathrm{OR}=2.42,0.76-6.61)$. These odds ratios can be interpreted as the single retrospective cohort study (Ferley et al. 1989) reported odds ratios that were 2.42 times greater than odds ratios reported from other study design types, and that studies that adjusted for gender reported odds ratios that were 2.42 times greater than odds 


\begin{tabular}{|c|c|c|c|c|c|}
\hline \multicolumn{2}{|l|}{ Freshwater Indicators: } & & \multirow{2}{*}{$\begin{array}{c}\text { Odds Ratio [95\% Cl] } \\
3.13[2.21,4.44] \\
4.90[3.08,7.80] \\
1.57[0.88,2.80]\end{array}$} & P-Value & \multirow{2}{*}{$\begin{array}{l}\text { Studies } \\
\text { [1], [2] } \\
\text { [2] }\end{array}$} \\
\hline $\begin{array}{l}\text { Total Coliform }<=10,000 \mathrm{cfu} / 100 \mathrm{~mL}{ }^{1} \\
\text { Total Coliform }>10,000 \mathrm{cfu} / 100 \mathrm{~mL} \\
\text { Total Coliform ROR }\end{array}$ & $\begin{array}{l}\text { (3 Sites) } \\
\text { (1 Site) }\end{array}$ & + & & 0.10 & \\
\hline $\begin{array}{l}\text { Fecal Coliform }<=200 \mathrm{cfu} / 100 \mathrm{~mL} \\
\text { Fecal Coliform }>200 \mathrm{cfu} / 100 \mathrm{~mL} \\
\text { Fecal Coliform ROR }\end{array}$ & $\begin{array}{l}\text { (5 Sites) } \\
\text { (4 Sites) }\end{array}$ & $\stackrel{+}{+}$ & $\begin{array}{l}2.05[1.19,3.54] \\
3.47[2.40,5.02] \\
1.69[0.88,3.27]\end{array}$ & 0.12 & $\begin{array}{l}\text { [1], [2], [3], [4], [5] } \\
\text { [6], [2], [3] }\end{array}$ \\
\hline $\begin{array}{l}\text { E. coli }<=126 \mathrm{cfu} / 100 \mathrm{~mL}^{3} \\
\text { E. coli }>126 \mathrm{cfu} / 100 \mathrm{~mL} \\
\text { E. coli ROR }\end{array}$ & $\begin{array}{l}\text { (1 Sites) } \\
\text { (2 Sites) }\end{array}$ & + & $\begin{array}{c}5.62[0.26,120] \\
3.46[2.24,5.35] \\
0.62[0.03,13.55]\end{array}$ & 0.38 & $\begin{array}{l}\text { [18] } \\
{[4],[19]}\end{array}$ \\
\hline \multicolumn{6}{|l|}{ Marine Indicators: } \\
\hline $\begin{array}{l}\text { Total Coliform }<=10,000 \mathrm{cfu} / 100 \mathrm{~mL}{ }^{4} \\
\text { Total Coliform }>10,000 \mathrm{cfu} / 100 \mathrm{~mL} \\
\text { Total Coliform ROR }\end{array}$ & $\begin{array}{l}\text { (6 Sites) } \\
\text { (3 Sites) }\end{array}$ & + & $\begin{array}{l}1.23[1.00,1.51] \\
2.29[1.56,3.35] \\
1.86[1.21,2.87]\end{array}$ & 0.005 & $\begin{array}{l}\text { [7], [8], [9], [10], [17] } \\
\text { [12], [13], [17] }\end{array}$ \\
\hline $\begin{array}{l}\text { Fecal Coliform }<=400 \mathrm{cfu} / 100 \mathrm{~mL}^{5} \\
\text { Fecal Coliform }>400 \mathrm{cfu} / 100 \mathrm{~mL} \\
\text { Fecal Coliform ROR }\end{array}$ & $\begin{array}{l}\text { (7 Sites) } \\
\text { (11 Sites) }\end{array}$ & + & $\begin{array}{l}1.28[1.01,1.63] \\
1.86[1.44,2.41] \\
1.45[1.02,2.07]\end{array}$ & 0.039 & $\begin{array}{l}\text { [14], [7], [8], [10], [15] } \\
\text { [14], [12], [8], [9], [13] }\end{array}$ \\
\hline $\begin{array}{l}\text { E. coli }<=35 \mathrm{cfu} / 100 \mathrm{~mL}^{6} \\
\text { E. coli }>35 \mathrm{cfu} / 100 \mathrm{~mL} \\
\text { E. coli ROR }\end{array}$ & $\begin{array}{l}\text { (3 Sites) } \\
\text { (19 Sites) }\end{array}$ & + & $\begin{array}{l}1.36[1.07,1.74] \\
2.70[2.19,3.34] \\
1.98[1.43,2.75]\end{array}$ & 0.001 & $\begin{array}{l}\text { [8], [16] } \\
\text { [14], [16], [13] }\end{array}$ \\
\hline $\begin{array}{l}\text { Enterococci }<=35 \mathrm{cfu} / 100 \mathrm{~mL} * 7 \\
\text { Enterococci }>35 \mathrm{cfu} / 100 \mathrm{~mL} \\
\text { Enterococci ROR }\end{array}$ & $\begin{array}{l}\text { (3 Sites) } \\
\text { (20 Sites) }\end{array}$ & + & $\begin{array}{l}1.30[0.99,1.69] \\
2.65[1.92,3.66] \\
2.04[1.34,3.09]\end{array}$ & 0.001 & $\begin{array}{l}\text { [14], [8] } \\
\text { [14], [8], [13], [10], [16], [15] }\end{array}$ \\
\hline $\begin{array}{l}\text { Streptococci }<=35 \mathrm{cfu} / 100 \mathrm{~mL}^{8} \\
\text { Streptococci > } 35 \mathrm{cfu} / 100 \mathrm{~mL} \\
\text { Streptococci ROR }\end{array}$ & $\begin{array}{l}\text { (4 Sites) } \\
\text { (10 Sites) }\end{array}$ & + & $\begin{array}{l}1.32[1.05,1.66] \\
2.25[1.51,3.36] \\
1.70[1.07,2.71]\end{array}$ & 0.024 & $\begin{array}{l}\text { [14], [7], [8] } \\
{[14],[12],[9]}\end{array}$ \\
\hline
\end{tabular}

Fig. 1 Summary of meta-analysis results, odds ratios, and Ratio of Odds Ratios (ROR). Number of individual sites with reported odds ratios is given next to each indicator, as well as the number of total studies included in each subanalysis in the Studies column. Footnotes: Meta-analysis summary results.

1. total coliform cut-off: San Diego Water Board (2007)

2. fecal coliform cut-off: California Department of Public Health (2000)

3. E. coli cut-off: U.S. EPA (1986)

4. total coliform cut-off: California State Water Resources Control Board (1990)

5. fecal coliform cut-off: California State Water Resources Control Board (1990)

6. E. coli cut-off: Haile et al. (1999)

7. enterococcus cut-off: U.S. EPA (1986)

8. streptococci cut-off: Ogan (1994)

9. ROR is the ratio of odds ratios from high vs. low indicator settings

[1] Lee et al. (2002), [2] Ferley et al. (1989), [3] Fewtrell et al. (1992), [4] Medema et al. (1995), [5] Seyfried et al. (1985), [6] Dewailly et al. (1986), [7] Jones et al. (1991), [8] Cabelli et al. (1979), [9] Alexander et al. (1992), [10] Colford et al. (2007), [11] Wade et al. (2008), [12] Prieto et al. (2001), [13] Haile et al. (1999), [14] Cheung et al. (1990), [15] Von Schirnding et al. (1992), [16] Cabelli (1983), [17] Dwight et al. (2004), [18] Van Asperen et al. (1997), [19] Wiedenmann et al. (2006), [20] Charoenca and Fujioka (1995)

ratios reported from studies that did not adjust for gender. For fecal streptococcus, the main source of heterogeneity was study size $(\mathrm{OR}=1.000041,95 \%$ CI 0.99 1.00009).

For marine settings, the primary sources of heterogeneity in the meta-analysis considering $E$. coli were adjustment for visitor/native status of the study participants $(\mathrm{OR}=1.80$, 95\% CI $1.13-2.88)$ and ethnicity $(0.54,95 \%$ CI $0.28-1.07)$. For enterococci, the main contributors to heterogeneity were adjustment for visitor/native status $(\mathrm{OR}=2.10,95 \% \mathrm{CI}$ 1.09-4.06), exposure category below or above $35 \mathrm{cfu} /$ $100 \mathrm{~mL}(\mathrm{OR}=1.38,95 \%$ CI $0.84-2.27)$, gender $(\mathrm{OR}=$ $0.17,95 \%$ CI $0.07-0.46)$, socioeconomic status $(\mathrm{OR}=5.66$, 95\% CI 1.53-20.8), and age $(\mathrm{OR}=0.36,95 \%$ CI 0.20 , $0.67)$.
Publication bias

A statistical test for publication bias (Begg and Mazumdar 1994) suggested that for some marine indicators, publication bias may have been present (marine fecal streptococci $=$ 0.001 , marine fecal coliform $p=0.017$, marine enterococci $p=0.001$, marine E. coli $p=0.001$, marine Klebsiella $p=0.003$, marine $P$. aeruginosa $p=0.009$, marine staphylococci $p=0.08)$. The Begg test plots study effect size against a measure of the study's standard error or sample size, and determines if the study effect sizes are symmetrically distributed around the overall summary effect. If these plots are not symmetrically distributed, it is likely that publication bias may be present. This suggests that the summary relative risks reported in this study may be overestimates. 
A further analysis was done using the "trim and fill" method proposed by Duval and Tweedie (2000), which nonparametrically attempts to account for the effects of publication bias and create an unbiased summary effect estimate (Duval and Tweedie 2000). For fecal streptococci in marine settings, publication bias was suspected in studies with indicator levels greater than $35 \mathrm{cfu} / 100 \mathrm{~mL}$. The "trim and fill" analysis gave a random effects OR of 1.97 (95\% CI 1.37, 2.84) for studies with indicator levels greater than $35 \mathrm{cfu} / 100 \mathrm{~mL}$ (previous unadjusted summary OR was 2.25, 95\% CI 1.51-3.36). The ROR for marine fecal streptococci, adjusted for publication bias, becomes 1.49 (95\% CI 0.97-2.30). For marine fecal coliforms, after adjusting for potential publication bias, the new ROR is 1.33 (95\% CI $0.95,1.88)$. For marine enterococci, the adjusted ROR was 1.31 (95\% CI 0.86, 1.98). For marine E. coli, the ROR adjusted for publication bias was 1.76 (95\% CI 1.22, 2.54). For marine Klebsiella, no ROR was calculated, but an adjusted summary OR was calculated to be 1.38 (95\% CI 1.1, 1.74). Marine $P$. aeruginosa also had no ROR to adjust, but the adjusted OR was 1.36 (95\% CI 1.12, 1.65). For marine staphylococci, the adjusted OR was 1.80 (95\% CI 1.26, 2.56).

\section{Discussion}

There are several microbiological indicators that are associated with skin-related health conditions in marine waters. This review has provided some evidence that skin-related health conditions are associated with exposure to contaminated recreational waters. All marine indicators showed statistically significant associations, with enterococci demonstrating the strongest association between bacterial levels and skin symptoms ( $\mathrm{ROR}=2.04,95 \%$ CI 1.34-3.09). Cutoff points for freshwater indicators did not demonstrate statistically significant associations with skin-related outcomes, but were suggestive of an association. However, this review found that few published studies have examined indicator organisms and skin-related outcomes in freshwater situations. The small number of freshwater studies is probably an important factor in the lack of significant findings for freshwater indicators. For the freshwater analyses, the number of study sites per indicator ranged from a low of three to a high of nine sites. However, for marine studies, the minimum number of sites for any one indicator was nine sites, and the maximum was twenty.

For these indicators, predefined cut-off points were used. Other cut-off points may have been chosen that would have maximized the ROR, but such data exploration would have to be accounted for with penalized p-values for multiple comparisons. Additionally, looking cut-off points that would maximize the risk would be better answered with primary study data, rather than in a meta-analysis setting which suffers from more potential biases than individual studies.
Skin ailments (rashes, skin infections and irritation) among swimmers could arise from a wide variety of causes ranging from physical irritation to actual infection. However, since our review observed a higher rate of skin ailments at marine sites with higher levels of fecal contamination, a cause independent of physical irritation is implied. Skin ailments among swimmers may be caused by a wide variety of pathogenic microorganisms, some of which would be naturally occurring and not necessarily expected to be associated with fecal indicator bacteria (cyanobacteria, cercarial dermatitis, sea-bather's eruption caused by zooplankton) (Burke 2002). However, other pathogens such as Pseudomonas, Staphylococcus, and adenovirus that could cause skin irritations could co-occur with fecal indicators associated with run-off, sewage discharge or through the shedding of other swimmers (CDC 2008).

The meta-regressions provided interesting information that could prove valuable for future studies in recreational water. The results from the meta-regressions indicate that there is evidence that controlling for native or visitor status of study participants may be an important factor to consider in future studies. The marine studies that used E. coli and adjusted for visitor/native status reported an odds ratio that was 1.80 times greater compared to studies that did not adjust (95\% CI 1.13-2.88), and studies that adjusted for ethnicity had odds ratios that were 0.54 times smaller than studies that did not (95\% CI 0.28-1.07). Thus, both native/visitor status and ethnicity appear to be important covariates to consider for adjustment. Studies of enterococci also supported the finding that visitor/native status was an important variable to adjust for $(\mathrm{OR}=2.10,95 \%$ CI 1.09-4.06), but other explanations of heterogeneity included exposure category below or above $35 \mathrm{cfu} / 100 \mathrm{~mL}(\mathrm{OR}=1.38,95 \%$ CI 0.84-2.27), gender $(\mathrm{OR}=0.17,95 \% \mathrm{CI} 0.07-0.46)$, socioeconomic status $(\mathrm{OR}=5.66,95 \%$ CI 1.53-20.8), and age $(\mathrm{OR}=0.36$, 95\% CI 0.20, 0.67).

Other possible sources of heterogeneity are indicated in the freshwater meta-analyses. Among studies that examined fecal coliforms as an indicator, the single retrospective cohort study (Ferley et al. 1989) reported an odds ratio that was 2.42 times greater than studies that did not use the retrospective cohort design (95\% CI 1.08-5.41). For studies examining fecal streptococcus, it appeared that larger studies tended to report larger odds ratios. For a 1,000-person increase in study size, the odds ratio increased by a factor of 1.04 times (95\% CI 0.99-1.09, p-value 0.08).

\section{Biases}

Publication bias was also seen in several of the subanalyses. Analysis of marine indicators indicated that with the exception of total coliforms, publication bias was present for the rest of the indicators. For fecal streptococci, fecal coliforms, and marine enterococci, after adjusting for publication bias 
Fig. 2 Studies with internal control groups (OR comparing swimmers to non-swimmers in water with lower than recommended indicator levels versus OR comparing swimmers to non-swimmers in water with higher than recommended indicator levels). Each study has a ROR reported, and if multiple studies are present for a given indicator, the RORs from each individual study are meta-analyzed into a summary ROR

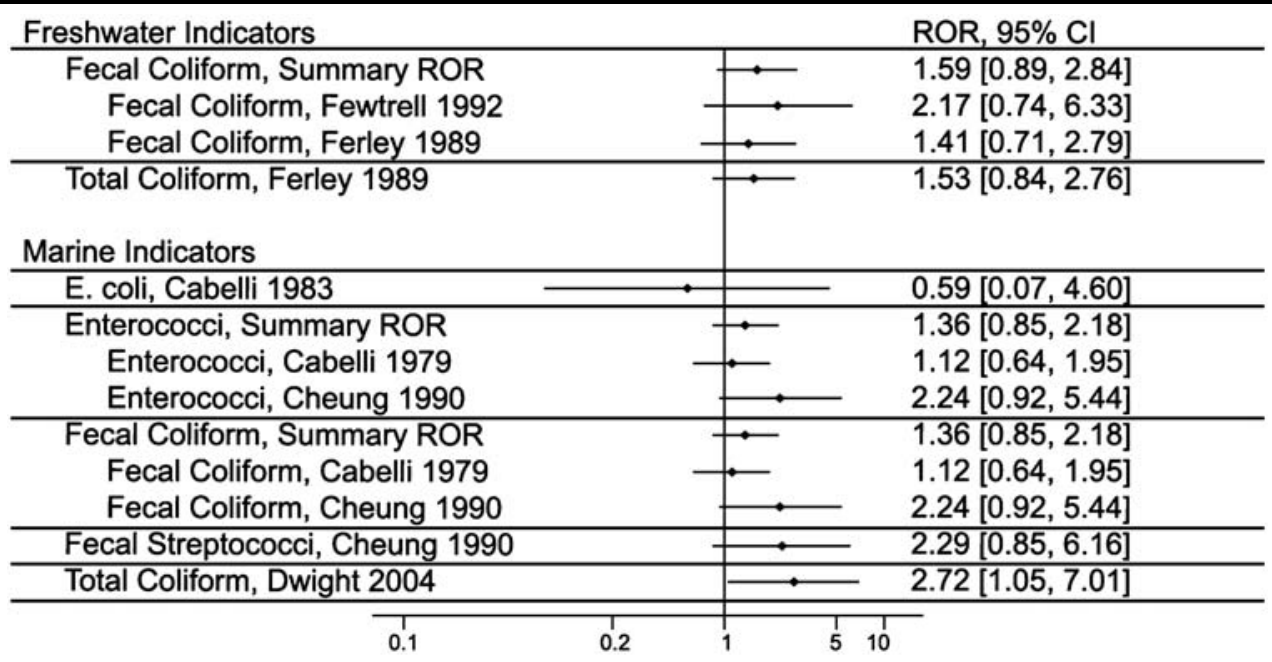

with the trim and fill method, the summary RORs changed from significant findings to non-significant findings. However, the ROR for marine E. coli remained significant. While this might cast doubt on the usefulness of fecal streptococci, fecal coliforms, and enterococci as indicator organisms, the direction of the ROR still indicates an association between indicator concentration and risk of skin conditions in bathers. However, these findings reinforce the idea that publication bias tends to overstate the association between indicator concentrations and the risk of skin disease.

Another source of bias was reported by (Fleisher and Kay 2006). It was found that bathers who perceived that there was a health risk associated with bathing in marine waters reported significantly higher rates of skin ailments compared to bathers who did not recognize any health risk associated with bathing in marine waters. Only one study in this systematic review adjusted for this variable (Haile et al. 1999). Thus, there is the potential for participants in other studies to have over-reported their incidence of skin ailments, thus theoretically causing results in those studies to be biased upwards.

Another potential source of bias is the comparison of swimmers to non-swimmers. These populations may have inherent differences that might confound the association between indicators and skin-related outcomes. For example, swimmers might be healthier individuals while nonswimmers were more prone to illness, or perhaps individuals with higher SES might be better educated about the risks associated with swimming, while those with lower SES might be more willing to swim and become exposed. Also, swimmers might be more likely to report symptoms than nonswimmers because they suspected that swimming may have caused whatever symptoms they experienced.

Yet another potential source of bias is that some studies relied on individuals to self-report their exposure and outcome status after the study. Swimmers might have poor recall of their exact exposure status, and they may have been more likely to report symptoms if they knew they had been exposed for long periods of time. This form of recall bias may have been present in many studies, because few were able to assign defined swimming activities and times to study participants or to employ physicians to assess outcome status in a blinded fashion.

In order to minimize biases associated with comparing disparate study populations, another analysis was conducted calculating ROR measures for studies with internal controls (an OR for high indicator concentration swimmers vs. nonswimmers and an OR for low indicator concentration swimmer vs. non-swimmers). Nine out of ten studies with internal controls demonstrated an elevated odds ratio for skinassociated outcomes in more polluted waters compared to less polluted waters, though only one was statistically significant (Fig. 2).

An alternative way to deal with the biases present in various studies is to assign different weights to different studies, with more rigorous and high quality studies receiving more weight while smaller and potentially more biased studies receiving lower weights. While weighting schemes were considered, the method of assigning weights is very subjective. Without a standard, systematic method of assigning weights, the results of this study might be skewed to indicate that a certain indicator was worse or better than the raw data suggests. Rather than weight the data, the authors chose to allow the readers to look at the data themselves and draw their own conclusions.

\section{Suggested further research}

It is evident that the current state of the freshwater indicator literature with respect to skin-related health outcomes is lacking. Future studies should continue to consider skinassociated outcomes using both traditional and novel indicators of recreational water quality. Another issue that should be explored would be for studies to use a measure of bather density and determine if it has any influence on health re- 


\section{Freshwater Indicators:}

Total Coliform $<=10,000 \mathrm{cfu} / 100 \mathrm{~mL}$ (3 Sites) Total Coliform > 10,000 cfu / $100 \mathrm{~mL}$

Fecal Coliform $<=200 \mathrm{cfu} / 100 \mathrm{~mL}$ Fecal Coliform > $200 \mathrm{cfu} / 100 \mathrm{~mL}$

E. coli $<=126 \mathrm{cfu} / 100 \mathrm{~mL}$

E. coli $>126 \mathrm{cfu} / 100 \mathrm{~mL}$

Enterococcus $>33 \mathrm{cfu} / 100 \mathrm{~mL}$

Staphylococci (n/a cut-point)

Streptococci (n/a cut-point)
(1 Site)

(5 Sites)

(4 Sites)

(1 Sites)

(2 Sites)

(3 Sites)

(4 Sites)

(7 Sites)

$\begin{array}{ccc}\begin{array}{c}\text { Indicator } \\ \text { Mean }\end{array} & \begin{array}{c}\text { Concentration } \\ \text { Median }\end{array} & \begin{array}{c}100 \mathrm{~mL} \text { ) } \\ \text { Range }\end{array} \\ 1079 & 786 & 10-2440 \\ 2446 & 24461 & 24461-24461 \\ 69 & 76 & 6-133 \\ 889 & 762 & 285-1749 \\ 68 & 68 & 68-68 \\ 153 & 153 & 136-170 \\ 297 & 83 & 37-770 \\ 45 & 13 & 3-151 \\ 31 & 15 & 13-82\end{array}$

\section{Marine Indicators:}

Total Coliform <= $10,000 \mathrm{cfu} / 100 \mathrm{~m}$
Total Coliform > $10,000 \mathrm{cfu} / 100 \mathrm{~m}$
Fecal Coliform <= $400 \mathrm{cfu} / 100 \mathrm{~m}$
Fecal Coliform > $400 \mathrm{cfu} / 100 \mathrm{~mL}$
E. coli <= $35 \mathrm{cfu} / 100 \mathrm{~mL}$
E. coli $>35 \mathrm{cfu} / 100 \mathrm{~mL}$
Enterococci <=35 cfu / $100 \mathrm{~mL}$
Enterococci > $35 \mathrm{cfu} / 100 \mathrm{~mL}$
Streptococci <= $35 \mathrm{cfu} / 100 \mathrm{~mL}$
Streptococci > 35 cfu / $100 \mathrm{~mL}$
Klebsiella (n/a cut-point)
P. aeruginosa (n/a cut-point)
Staphylococci (n/a cut-point)

$\begin{array}{lc}\text { (6 Sites) } & 744 \\ \text { (3 Sites) } & 10667 \\ \text { (7 Sites) } & 95 \\ \text { (11 Sites) } & 797 \\ \text { (3 Sites) } & 11 \\ \text { (19 Sites) } & 1311 \\ \text { (4 Sites) } & 22 \\ \text { (21 Sites) } & 1250 \\ \text { (4 Sites) } & 21 \\ \text { (10 Sites) } & 159 \\ \text { (11 Sites) } & 205 \\ \text { (13 Sites) } & 11 \\ \text { (11 Sites) } & 952\end{array}$

574
10000
77
565
15
269
28
168
24
101
105
5
921

37-2022

10000-12000

8-254

400-3166

$2-15$

35-10400

7-31

$35-9160$

4-32

40-500

4-943

$0.07-46$

100-2963

Fig. 3 Microbial indicator concentrations by indicator. Mean, median, and minimum/maximum values for each microbial indicator are reported

lated outcomes. Bather density could be an important variable because higher bather densities might elevate the concentration of bacteria in the water by re-suspending sediments or shedding of indicators and/or pathogens.

\section{Limitations}

One of the major criticisms of meta-analyses of observational studies is that it is probable that there are biases and confounding factors that have not been adjusted for in the individual studies, and that the populations in each study are not comparable to populations in other studies (Shapiro 1994). This would make any summary measures suspect. In order to attempt to deal with the heterogeneity of the data, random-effects analyses were used whenever appropriate. There was also the possible bias that studies without significant findings may not have been published. Although every effort was made to obtain relevant studies, dissertations, and reports, some studies may not have been found, and some studies that were relevant may have not published enough data to extract because no significant findings were found.

Another limitation to consider is the vast difference between many of the study populations and sites. The studies included range across Asia (Lee et al. 2002; Cheung et al. 1990), Europe (Jones et al. 1991; Alexander et al. 1992; Fewtrell et al. 1992; Ferley et al. 1989; Medema et al. 1995; Prieto et al. 2001), Africa (Von Schirnding et al. 1992) and North America (Cabelli et al. 1979; Cabelli 1983; Seyfried et al. 1985; Dewailly et al. 1986; Haile et al. 1999; Dwight et al. 2004; Colford et al. 2007). Some studies specifically looked for tourists (Cabelli 1983) while other studies dealt with more native populations (Dwight et al. 2004).

\section{Conclusions}

The results of this review indicate that skin complaints may be significantly more likely to occur among swimmers exposed to marine waters with measured levels of total coliform, fecal coliform, E. coli, enterococci, and streptococci above the recommended cut-off points for these indicator organisms. No statistically significant results were found for freshwater cut-off points.

Open Access This article is distributed under the terms of the Creative Commons Attribution Noncommercial License which permits any noncommercial use, distribution, and reproduction in any medium, provided the original author(s) and source are credited. 


\section{Fresh E. coli- Cutoff: $<=126 / 100 \mathrm{~mL}$}

Study

10

10

Year

Van Asperen 1997

Subtotal (1-squared $=. \%, p=$.)

1

Medema $\quad 1995$

Wiecenmann 2006

Subtotal ( 1 squared $=0.0 \%, p=0.636$ )

Heterogeneity between groups: $p=0.759$

Overall (1-squared $=0.0 \%, p=0.853$ )

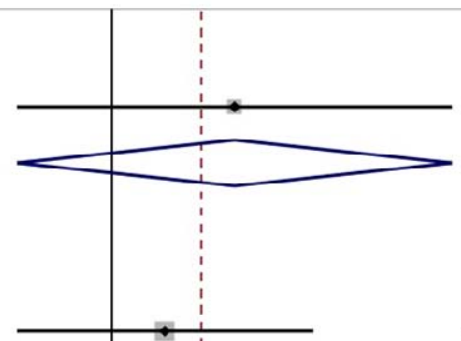

$2.11(0.26,17.14) \quad 4.24$

$3.54(2.27,5.52) \quad 93.78$

$3.46(2.24,5.35)$

$3.49(2.27,5.38)-100.00$

0.00

\section{Fresh Enterococcus- Cutoff: $<=33 / 100 \mathrm{~mL}$}

Study

ID
ES (95\% Cl)

Year

$\begin{array}{ll}1 & \\ \text { Wade } & \\ \text { van Asperen } & 1997 \\ \text { Wiedenmann } & 2006\end{array}$

Subtotal (I-squared $=86.1 \%, p=0.001$ )

Overall (1-squared $=86.1 \%, p=0.001)$

NOTE: Weights are from random effects aralysis
Weight

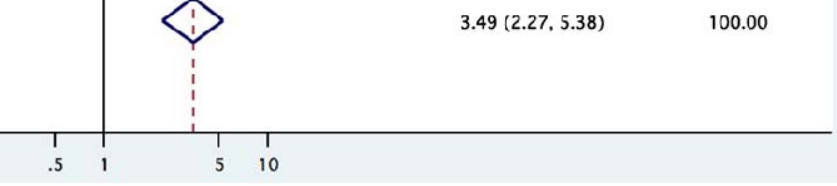




\section{Fresh Fecal Coliform- Cutpoint: $<=200 / 100 \mathrm{~mL}$}

Study

ID

Year

0

Lee 2002

Ferley $\quad 1989$

Fewtrell 1992

Medema 1995

Seyfried 1985

Subtotal ( $(1-$ squared $=35.3 \%, p=0.186$ )

.

1

Dewailly 1986

Ferley $\quad 1989$

Ferley 1989

Fewtrell 1992

Subtotal (I-squared $=32.1 \%, p=0.220$ )

.

Overall (I-squared $=47.6 \%, p=0.054$ )

NOTE: Weights are from random effects analysis

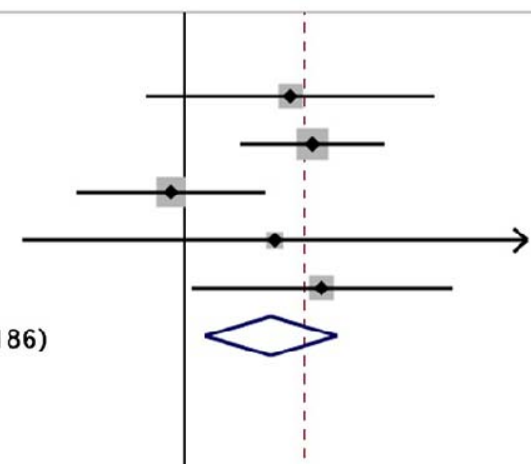

$2.40(0.73,7.90) \quad 6.66$

$2.90(1.59,5.30) \quad 14.94$

$0.90(0.41,1.96) \quad 11.54$

$2.11(0.26,17.14) \quad 2.61$

$3.12(1.06,9.19) \quad 7.68$

$2.05(1.19,3.54) \quad 43.43$

$3.33(1.17,9.51) \quad 8.00$

$4.90(3.08,7.80) \quad 18.08$

$3.40(2.14,5.40) \quad 18.14$

$1.95(0.94,4.08) \quad 12.35$

$3.47(2.40,5.02) \quad 56.57$

$2.72(1.90,3.88) \quad 100.00$

\section{Fresh Fecal Streptococci}

Study

ID Year

\begin{tabular}{|lc}
\hline Ferley & 1989 \\
\hline Ferley & 1989 \\
\hline Ferley & 1989 \\
\hline Fewtrell & 1992 \\
\hline Fewtrell & 1992 \\
\hline Medema & 1995 \\
\hline Seyfried & 1985 \\
\hline
\end{tabular}

Overall (I-squared $=60.3 \%, p=0.019$ )

NOTE: Weights are from random effects analysis
ES $(95 \%$ Cl) $\quad$ Weight

$4.90(3.08,7.80) \quad 20.15$

$3.40(2.14,5.40) \quad 20.20$

$2.90(1.59,5.30) \quad 17.34$

$1.95(0.94,4.08) \quad 14.83$

$0.90(0.41,1.96) \quad 14.02$

$2.11(0.26,17.14) \quad 3.61$

$3.12(1.06,9.19) \quad 9.85$

$2.65(1.73,4.07) \quad 100.00$ 


\section{Fresh Staphylococci}

Study

10

Year

ES $(95 \%$ a)

Fewtrell
Seyfried
Van Asperen
Overall $(1-$-squared $=33.0 \%, \mathrm{p}=0.214)$

\section{Fresh Total Coliform- Cutoff: $<=10000 / 100 \mathrm{~mL}$}

Study

ID

Year

0

Lee 2002

Ferley $\quad 1989$

Ferley 1989

Subtotal ( 1 -squared $=0.0 \%, p=0.829$ )

1

Ferley 1989

Subtotal $(\mathrm{I}$-squared $=. \%, \mathrm{p}=$.

Heterogeneity between groups: $p=0.130$

Overall ( $(1$-squared $=0.0 \%, p=0.446$ )
ES (95\% Cl) Weight

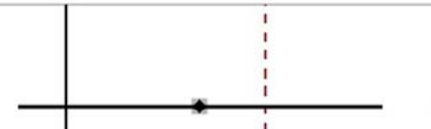

$2.40(0.73,7.90) \quad 5.52$

$3.40(2.14,5.40) \quad 36.63$

$2.90(1.59,5.30) \quad 21.56$

$3.13(2.20,4.44) \quad 63.72$

$4.90(3.08,7.80) \quad 36.28$

$4.90(3.08,7.80) \quad 36.28$

$3.68(2.78,4.87) \quad 100.00$ 


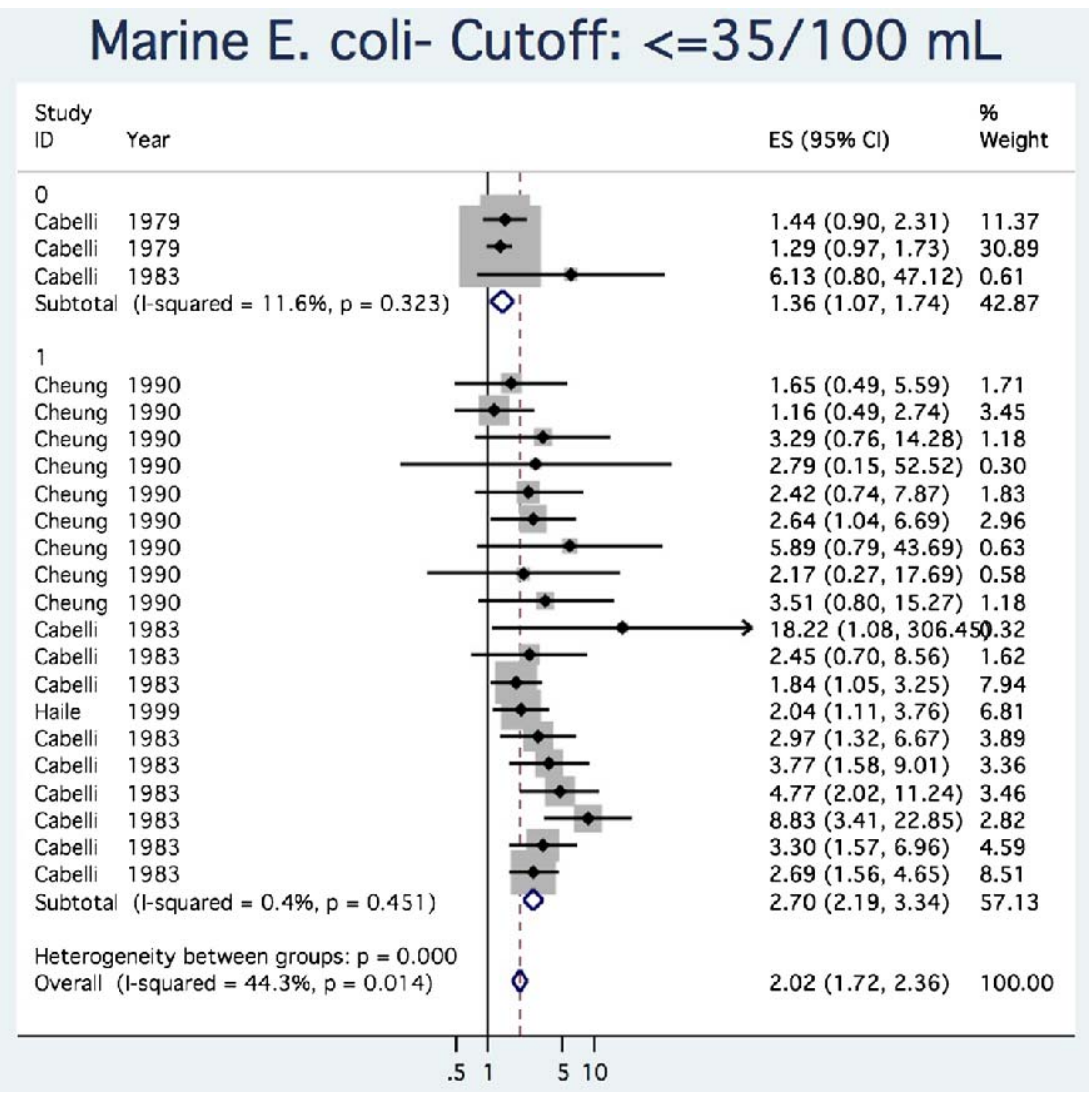

Marine Enterococci- Cutoff: $<=35 / 100 \mathrm{~mL}$

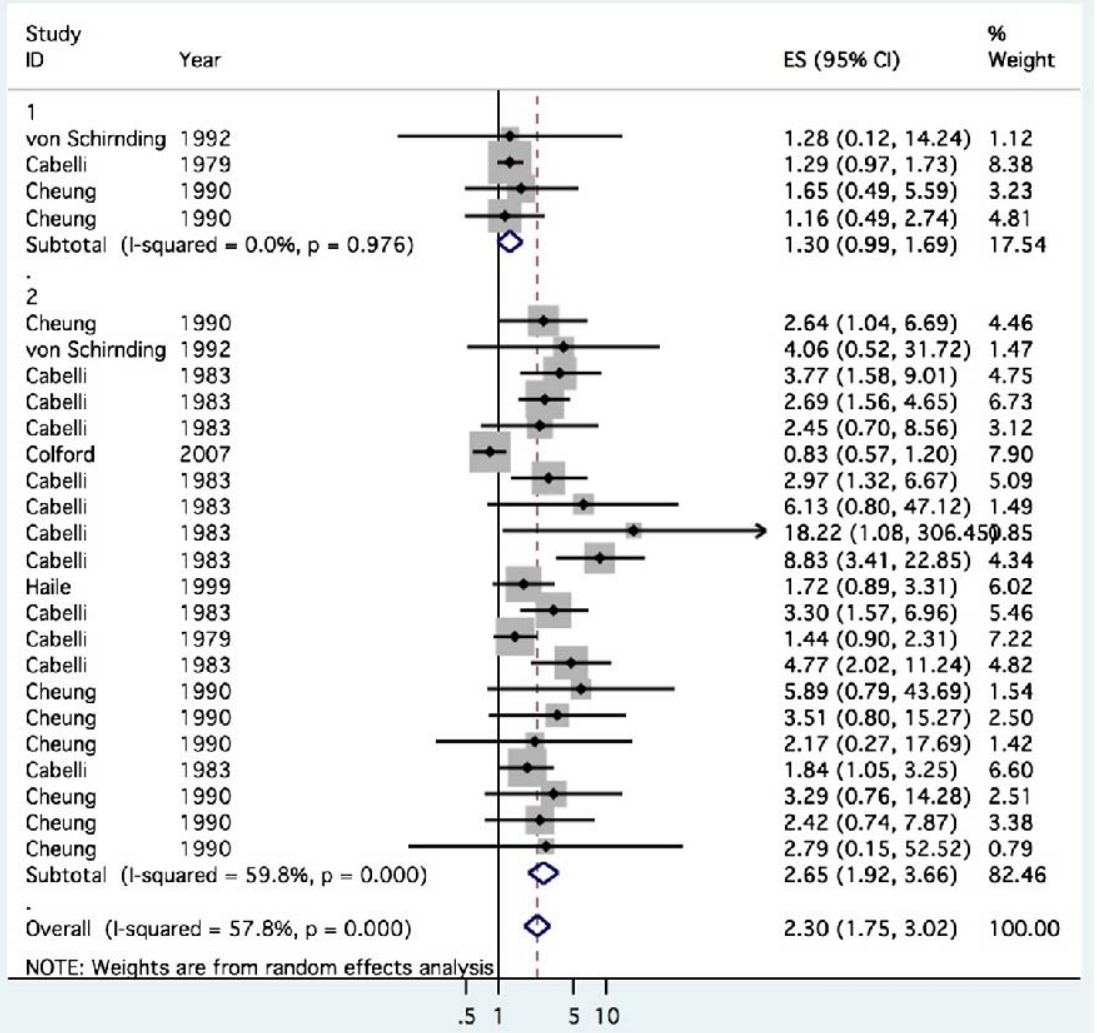


Marine Fecal Coliforms- Cutoff: $<=400 / 100 \mathrm{~mL}$

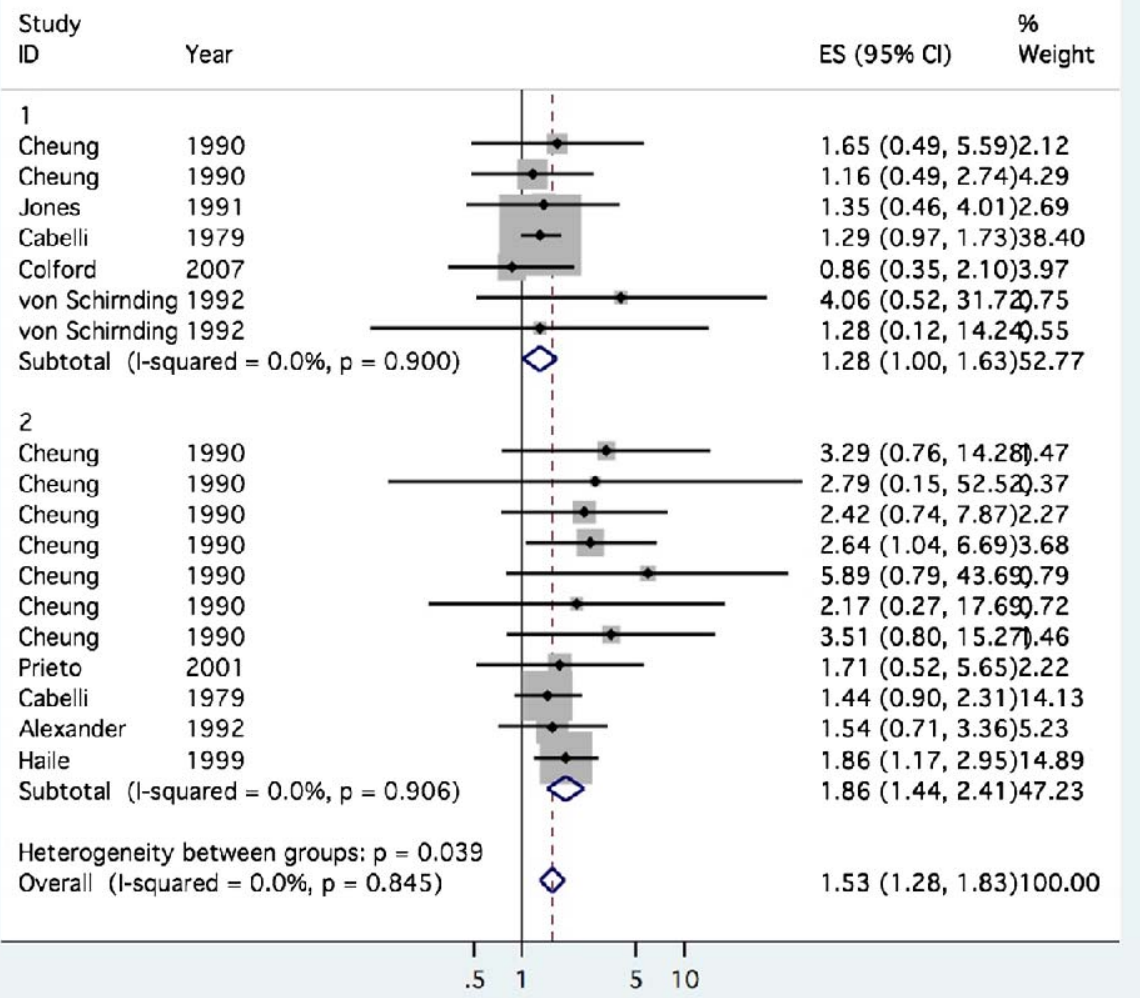

\section{Marine Fecal Strep- Cutoff: $<=35 / 100 \mathrm{~mL}$}

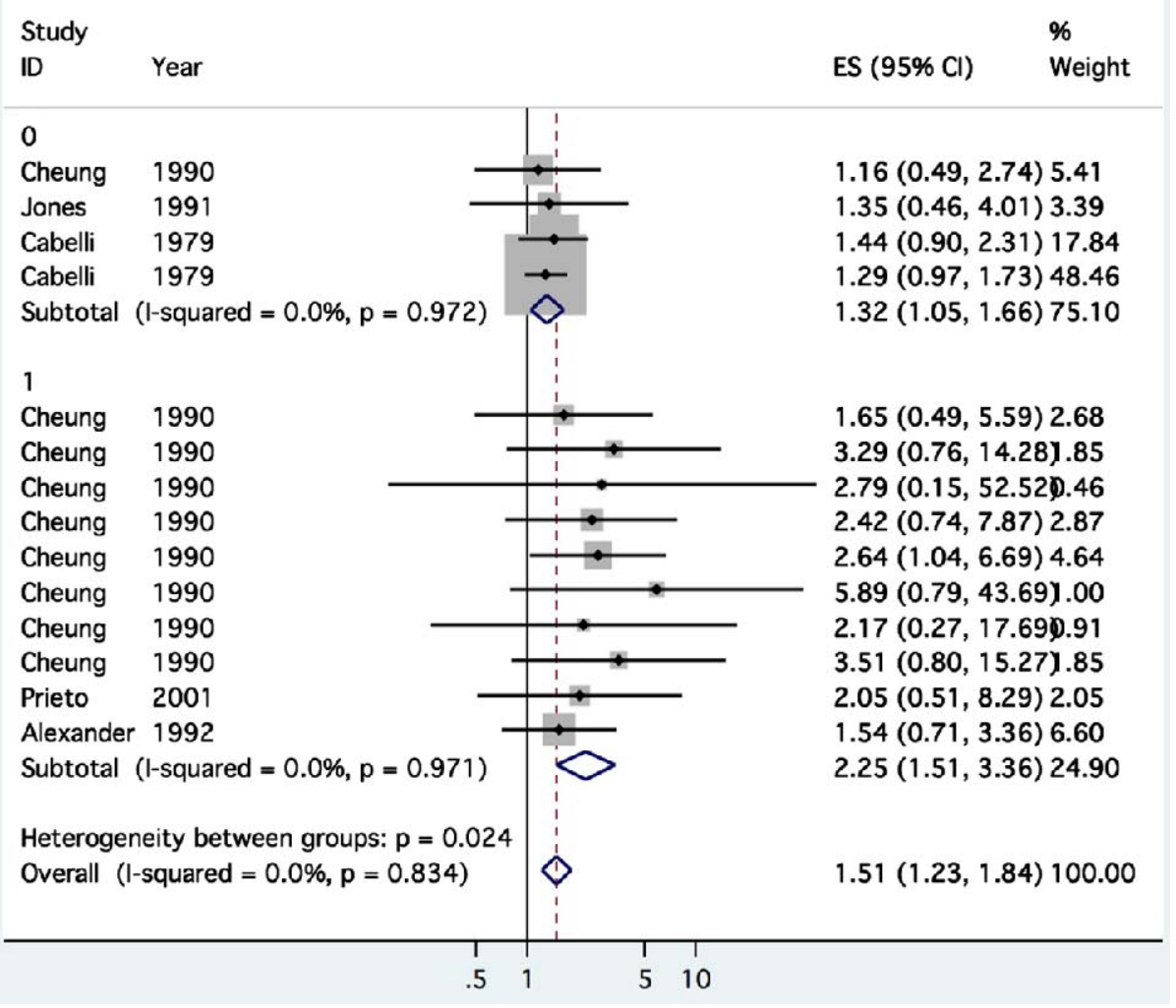


Marine Klebsiella

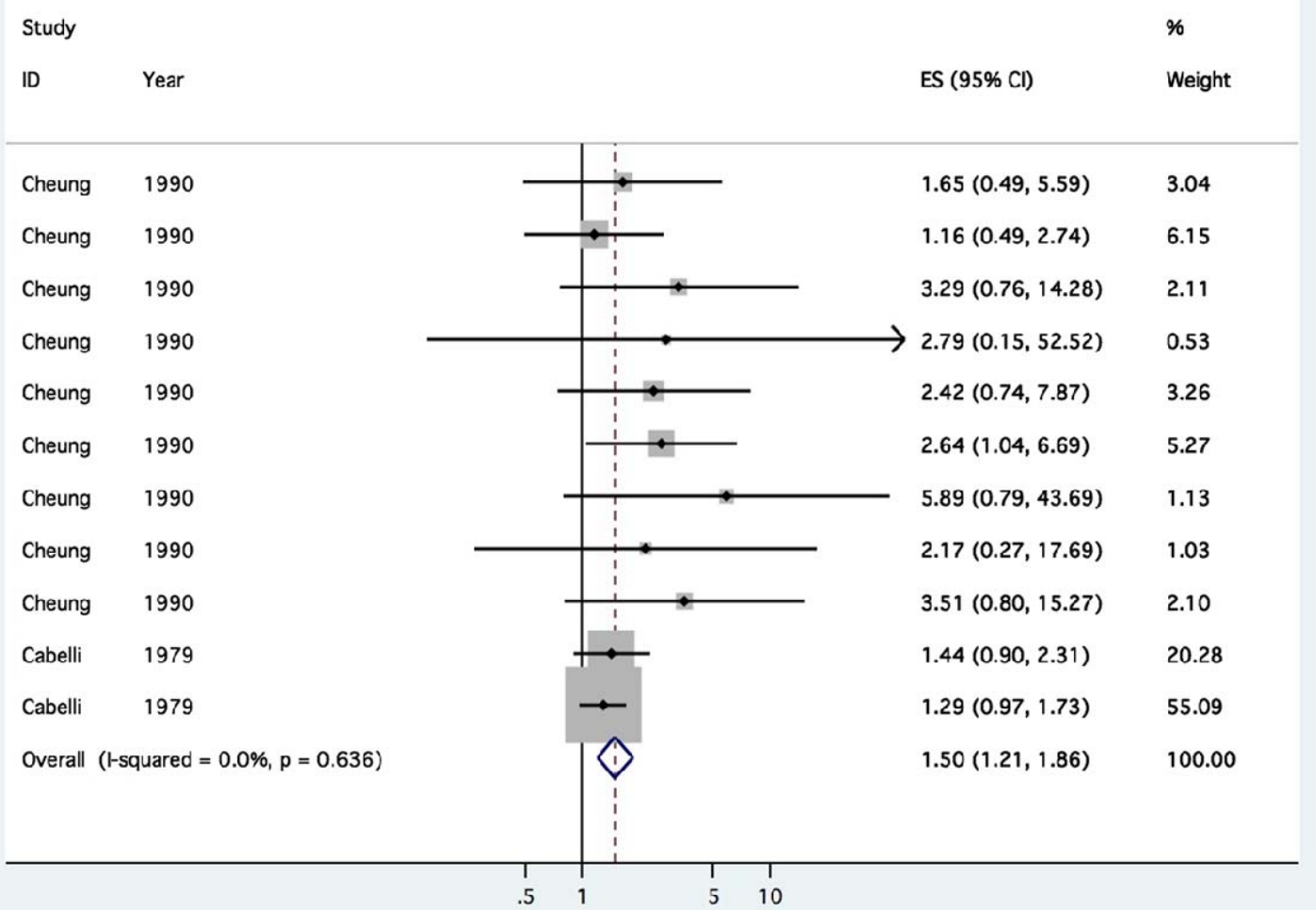

\section{Marine P. aeruginosa}

\begin{tabular}{|c|c|c|c|}
\hline \multicolumn{3}{|l|}{ Study } & \multirow{2}{*}{$\begin{array}{l}\% \\
\text { Weight }\end{array}$} \\
\hline ID & Year & ES $(95 \% \mathrm{Cl})$ & \\
\hline Cheung & 1990 & $1.65(0.49,5.59)$ & 2.85 \\
\hline Cheung & 1990 & $1.16(0.49,2.74)$ & 5.76 \\
\hline Cheung & 1990 & $3.29(0.76,14.28)$ & 1.97 \\
\hline Cheung & 1990 & $2.79(0.15,52.52)$ & 0.49 \\
\hline Cheung & 1990 & $2.42(0.74,7.87)$ & 3.05 \\
\hline Cheung & 1990 & $2.64(1.04,6.69)$ & 4.94 \\
\hline Cheung & 1990 & $5.89(0.79,43.69)$ & 1.06 \\
\hline Cheung & 1990 & $2.17(0.27,17.69)$ & 0.97 \\
\hline Cheung & 1990 & $3.51(0.80,15.27)$ & 1.96 \\
\hline Prieto & 2001 & $1.10(0.32,3.77)$ & 2.80 \\
\hline Jones & 1991 & $1.35(0.46,4.01)$ & 3.61 \\
\hline Cabelli & 1979 & $1.44(0.90,2.31)$ & 18.98 \\
\hline Cabelli & 1979 & $1.29(0.97,1.73)$ & 51.56 \\
\hline \multicolumn{2}{|c|}{ Overall $(I-$ squared $=0.0 \%, p=0.770)$} & $1.48(1.21,1.82)$ & 100.00 \\
\hline
\end{tabular}




\section{Marine Staphylococci}

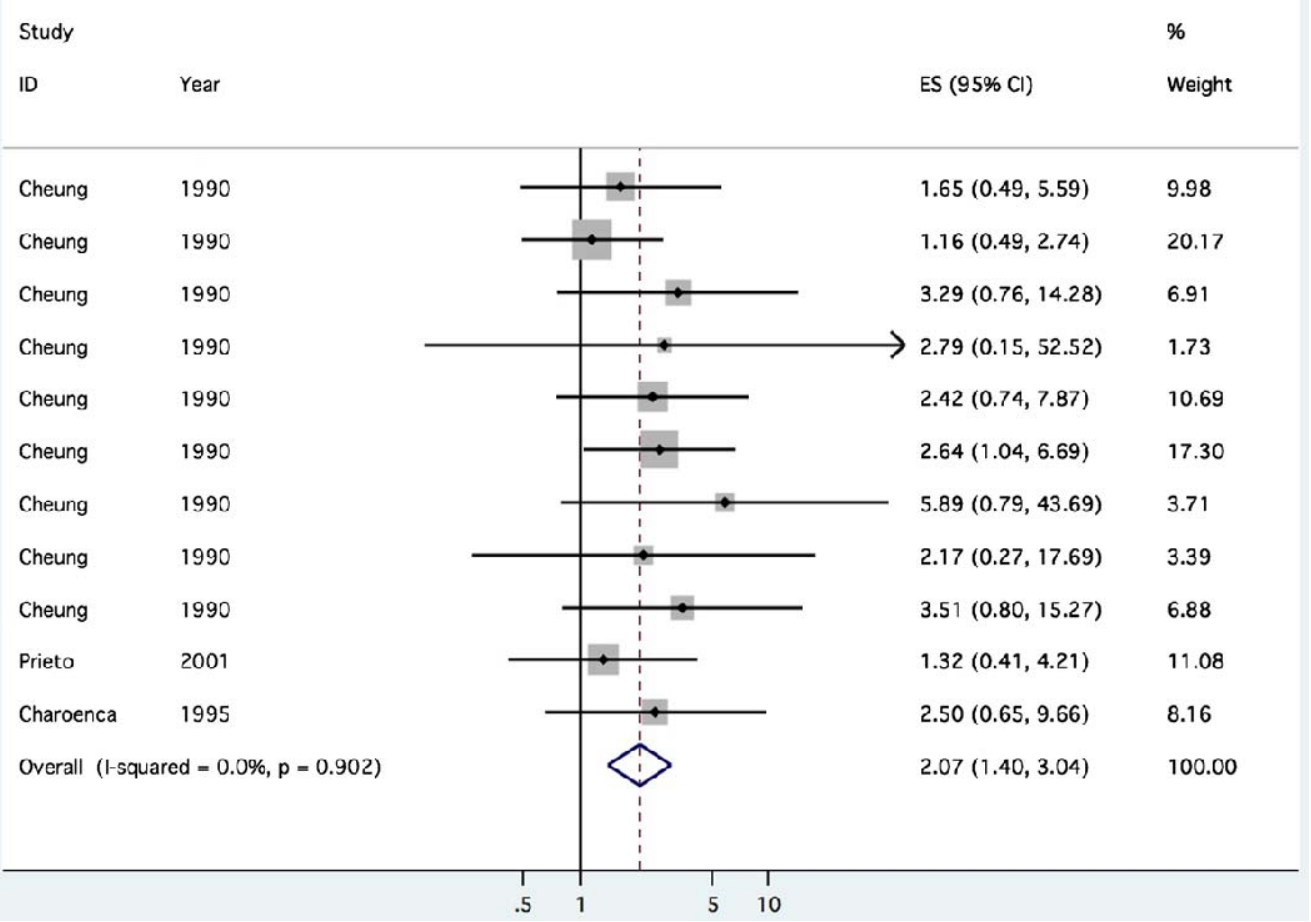

\section{Marine Total Coliform- Cutoff: $<=10,000 / 100 \mathrm{~mL}$}

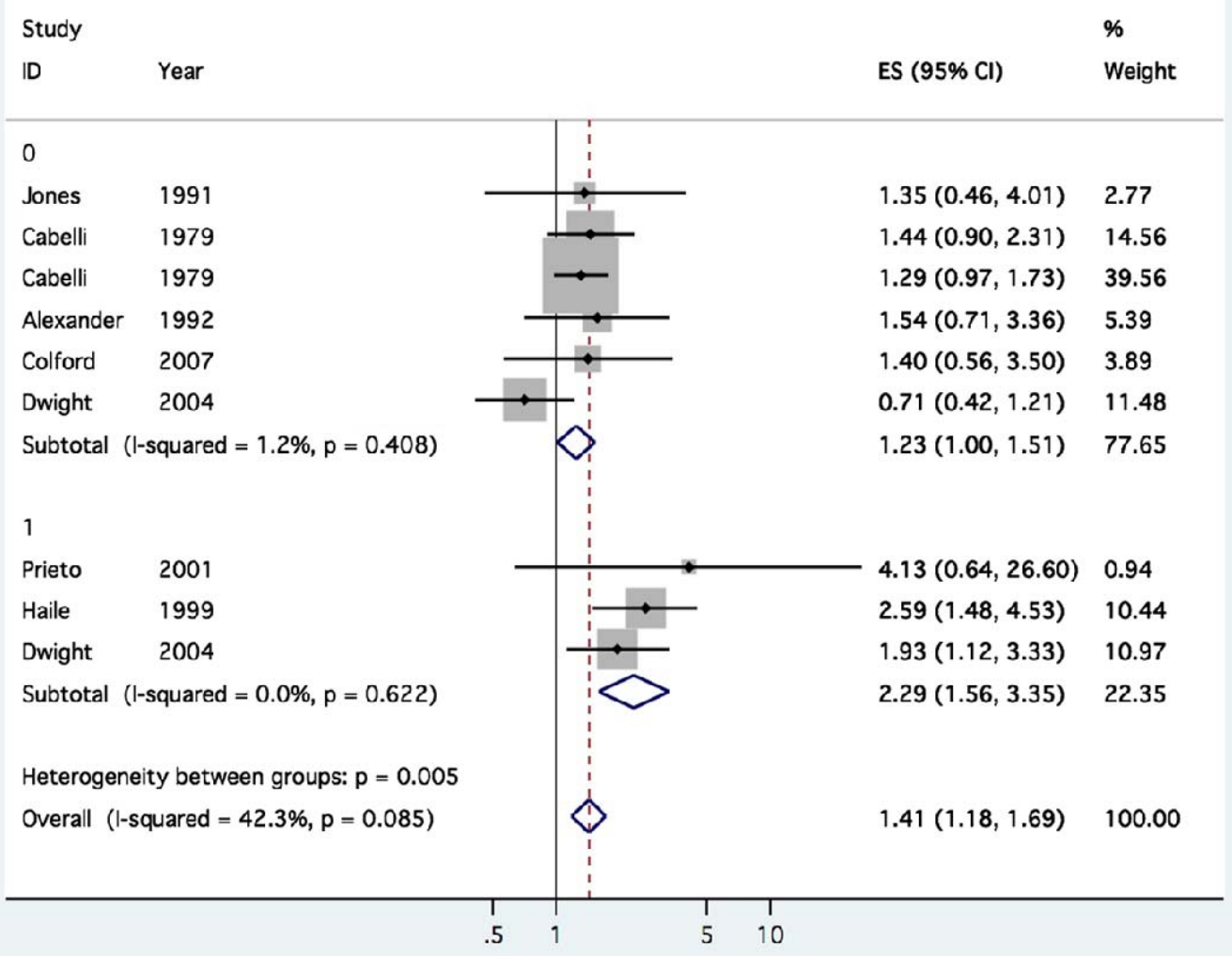




\section{References}

Alexander LM, Heaven A, Tennant A, Morris R (1992) Symptomatology of children in contact with sea water contaminated with sewage. J Epidemiol Community Health 46:340-344

Altman DG, Bland JM (2003) Interaction revisited: the difference between two estimates. Br Medical J 326:219

Amson JE (1991) Protection of divers in waters that are contaminated with chemicals or pathogens. Undersea Biomed Res 18:213-219

Balarajan R, Soni Raleigh V, Yuen P, Wheeler D, Machin D, Cartwright $\mathrm{R}$ (1991) Health risks associated with bathing in sea water. $\mathrm{Br}$ Medical J 303:1444-1445

Bandaranayake DT, McBride GB, Lewis GD, Till DG (1995) Health effects of bathing at selected New Zealand marine beaches. New Zealand

Baylet R, Sinegre F (1984) The role of sea-baths in the epidemiology of infectious diseases in the mediterranean coastal zone. Tech Sci Munic L'Eau 79:99-103

Begg CB, Mazumdar M (1994) Operating characteristics of a rank correlation test for publication bias. Biometrics 50:1088-1101

Bonilla TD, Nowosielski K, Cuvelier M, Hartz A, Green M, Esiobu N, McCorquodale DS, Fleisher JM, Rogerson A (2007) Prevalence and distribution of fecal indicator organisms in south Florida beach sand and preliminary assessment of health effects associated with beach sand exposure. Mar Pollut Bull 54:1472-1482

Burke W (2002) Skin problems related to noninfectious coastal microorganisms. Dermatol Ther 15:10-17

Cabelli V (1983) Health effects criteria for marine recreational waters. In: EPA, Research Triangle Park

Cabelli VJ, Dufour AP, Levin MA, McCabe LJ, Haberman PW (1979) Relationship of microbial indicators to health effects at marine bathing beaches. Am J Public Health 69:690-696

California Department of Public Health (2000) Appendix E - epidemiological studies related to ocean water and freshwater recreation. In: Health CDoP (ed)

California State Water Resources Control Board (1990) Water quality control plan for ocean waters of California (California Ocean Plan). State Board Resolution No. 90-27

California State Water Resources Control Board (1990b) Functional equivalent document: amendment of the water quality control plan for ocean waters of California (California Ocean Plan). State Board Resolution No. 90-27

CDC (2008) Morbidity and Mortality Weekly Report (MMWR) and Emerging Infectious Diseases (EID): skin, ear, and eye infections. http://www.cdc.gov/healthyswimming/mmwr.htm\#Skin

Charoenca N, Fujioka R (1995) Association of staphylococcal skin infections and swimming. Water Sci Technol 31:11-18

Cheung WH, Chang KC, Hung RP (1991) Variations in microbial indicator densities in beach waters and health-related assessment of bathing water quality. Epidemiol Infect 106:329-344

Cheung WH, Chang KC, Hung RP, Kleevens JW (1990) Health effects of beach water pollution in Hong Kong. Epidemiol Infect 105:139-162

Colford JM Jr, Wade TJ, Schiff KC, Wright CC, Griffith JF, Sandhu SK, Burns S, Sobsey M, Lovelace G, Weisberg SB (2007) Water quality indicators and the risk of illness at beaches with non-point sources of fecal contamination. Epidemiology 18:27-35

DerSimonian R, Laird N (1986) Meta-analysis in clinical trials. Control Clin Trials 7:177-187

Dewailly E, Poirier C, Meyer FM (1986) Health hazards associated with windsurfing on polluted water. Am J Public Health 76:690 691

Dufour AP (1984) Bacterial indicators of recreational water quality. Can J Public Health 75:49-56

Duval S, Tweedie R (2000) A nonparametric "trim and fill" method of accounting for publication bias in meta-analysis. JASA 95(449):89-98
Dwight RH, Baker DB, Semenza JC, Olson BH (2004) Health effects associated with recreational coastal water use: urban versus rural California. Am J Public Health 94:565-567

Fattal BP, Agursky T (1987) The association between seawater pollution as measured by bacterial indicators and morbidity among Mediterranean bathing beaches of Israel. Chemosphere 16:565570

Ferley JP, Zmirou D, Balducci F, Baleux B, Fera P, Larbaigt G, Jacq E, Moissonnier B, Blineau A, Boudot J (1989) Epidemiological significance of microbiological pollution criteria for river recreational waters. Int J Epidemiol 18:198-205

Fewtrell L, Godfree AF, Jones F, Kay D, Salmon RL, Wyer MD (1992) Health effects of white-water canoeing. Lancet 339:1587-1589

Fleisher JM, Kay D, Salmon RL, Jones F, Wyer MD, Godfree AF (1996) Marine waters contaminated with domestic sewage: nonenteric illnesses associated with bather exposure in the United Kingdom. Am J Public Health 86:1228-1234

Fleisher JM, Kay D (2006) Risk perception bias, self-reporting of illness, and the validity of reported results in an epidemiologic study of recreational water associated illnesses. Mar Pollut Bull 52:264268

Foulon G, Maurin J, Quoi N, Martin-Buoyer G (1983) Relationship between the microbiological quality of bathing water and health effects. Rev Francaise des Sciences de l'Eau 2:127-143

Haile RW (1996) An epidemiological study of possible adverse health effects of swimming in Santa Monica Bay. Final report

Haile RW, Witte JS, Gold M, Cressey R, McGee C, Millikan RC, Glasser A, Harawa N, Ervin C, Harmon P, Harper J, Dermand J, Alamillo J, Barrett K, Nides M, Wang G (1999) The health effects of swimming in ocean water contaminated by storm drain runoff. Epidemiology 10:355-363

Harrington JF, Wilcox DN, Giles PS, Ashbolt NJ, Evans JC, Kirton HC (1993) The health of Sydney surfers - an epidemiologic study. Water Sci Technol 27:175-181

Jones F, Kay D, Stanwellsmith R, Wyer M (1991) Results of the 1st pilot-scale controlled cohort epidemiologic investigation into the possible health-effects of bathing in seawater at Langland Bay, Swansea. J Inst Water Environ Manag 5:91-98

Kocasoy G (1989) The relationship between coastal tourism, sea pollution, and public health: a case study from Turkey. Environmentalist 9:245-251

Kueh CST, Lee T, Wong SL, Lloyd OL, Yu TS, Tam JS, Bassett DCJ (1995) Epidemiological study of swimming-associated illnesses relating to bathing-beach water quality. Water Sci Technol 31:1-

Lee J, Koh D, Andijani M, Saw SM, Munoz C, Chia SE, Wong ML, Hong CY, Ong CN (2002) Effluents from a pulp and paper mill: a skin and health survey of children living in upstream and downstream villages. Occup Environ Med 59:373-379

Marino FM, Martinez-Manzanares M, Borrego JJ (1995) Microbiological-epidemiological study of selected marine beaches in Malaga (Spain). Water Sci Technol 31:5-9

McBride GB, Salmond CE, Bandaranayake DR, Turner SJ, Lewis GD, Till DG (1998) Health effects of marine bathing in New Zealand. Int J Environ Health Res 8:173-189

Medema G, Van Asperen I, Klokman-Houweling J, Nooitgedagt A, Van de Laar M (1995) The relationship between health effects in triathletes and microbiological quality of freshwater. Water Sci Technol 31:19-26

New Jersey DoH (1988) New Jersey ocean health study final summary report. Final report

Ogan MT (1994) Improved recovery of fecal streptococci from tropical (Caribbean) marine recreational waters by anaerobic-MF method using m-enterococcus agar. Water, Air, and Soil Pollution 72:163177 
Philipp R, Evans EJ, Hughes AO, Grisdale SK, Enticott RG, Jephcott AE (1985) Health risks of snorkel swimming in untreated water. Int J Epidemiol 14:624-627

Pilotto LS, Douglas RM, Burch MD, Cameron S, Beers M, Rouch GJ, Robinson P, Kirk M, Cowie CT, Hardiman S, Moore C, Attewell RG (1997) Health effects of exposure to cyanobacteria (bluegreen algae) during recreational water-related activities. Aust N Z J Public Health 21:562-566

Prieto MD, Lopez B, Juanes JA, Revilla JA, Llorca J, DelgadoRodriguez M (2001) Recreation in coastal waters: health risks associated with bathing in sea water. J Epidemiol Community Health 55:442-447

Pruss A (1998) Review of epidemiological studies on health effects from exposure to recreational water. Int J Epidemiol 27:1-9

Robinton ED (1966) A quantitative and qualitative appraisal of microbial pollution of water by swimmers: a preliminary report. J Hyg Camb 64

San Diego WB (2007) Resolution and basin plan amendmentAppendix F, Water quality objectives for indicator bacteria. In: Board SDW (ed) California Environmental Protection Agency

Seyfried PL, Tobin RS, Brown NE, Ness PF (1985) A prospective study of swimming-related illness: I. Swimming-associated health risk. Am J Public Health 75:1068-1070

Seyfried PL, Tobin RS, Brown NE, Ness PF (1985b) A prospective study of swimming-related illness II. Morbidity and the microbiological quality of water. Am J Public Health 75:1071-1075

Shapiro S (1994) Meta-analysis/shmeta-analysis. Am J Epidemiol 140:771-778

StataCorp (2007) Stata statistical software: release 10. StataCorp LP, College Station

Stevenson A (1953) Studies of bathing water quality and health. Am J Public Health 43:529-538

Thompson SG, Sharp SJ (1999) Explaining heterogeneity in metaanalysis: a comparison of methods. Stat Medicine 18:2693-2708
U.S. EPA (1986) Bacteriological water quality criteria for marine and fresh recreational waters. EPA-440/5-84-002. Cincinnati, OH: U.S. Environmental Protection Agency, Office of Water Regulations and Standards

Van Asperen I, Medema G, Havelaar AH, Borgdorff MW (1997) Health effects of freshwater bathing among primary school children; Design for a randomised exposure study. Rijksinstituut voor Volksgezondheid en Milieu RIVM

Von Schirnding YE, Kfir R, Cabelli V, Franklin L, Joubert G (1992) Morbidity among bathers exposed to polluted seawater. A prospective epidemiological study. South Afr Medical J 81:543546

Wade TJ, Calderon RL, Brenner KP, Sams E, Beach M, Haugland R, Wymer L, Dufour AP (2008) High sensitivity of children to swimming-associated gastrointestinal illness: results using a rapid assay of recreational water quality. Epidemiology 19:375-383

Wade TJ, Pai N, Eisenberg JN, Colford JM Jr (2003) Do U.S. Environmental Protection Agency water quality guidelines for recreational waters prevent gastrointestinal illness? A systematic review and meta-analysis. Environ Health Perspect 111:1102-1109

Wiedenmann A, Kruger P, Dietz K, Lopez-Pila JM, Szewzyk R, Botzenhart K (2006) A randomized controlled trial assessing infectious disease risks from bathing in fresh recreational waters in relation to the concentration of Escherichia coli, intestinal enterococci, Clostridium perfringens, and somatic coliphages. Environ Health Perspect 114:228-236

World Health Organization (2001) Bathing water quality and human health: faecal pollution. Outcome of an expert consultation. World Health Organization, Farnham

Zmirou D, Ferley JP, Balducci F, Baleux B, Fera P, Larbaigt G, Jacq E, Moissonnier B, Blineau A, Boudot J (1990) Evaluation of microbial indicators of health risk related to river swimming places. Rev Epidemiol Sante Publique 38:101-110 